\title{
Limited Investigation of Auxiliary Tasking during Boundary Avoidance Tracking (AT BAT)
}

Ryan Blake, Maj, USAF

Project Manager/Test Pilot

Chun Hao Lee, Maj, TAF

Test Pilot

Chris Keithley, Capt, USAF

Test Pilot
Nancy Baccheschi, Capt, USAF Flight Test Engineer

Glenn Cooley, Capt, USAF

Flight Test Engineer

December 2008

\section{FINAL TECHNICAL INFORMATION MEMORANDUM}

Approved for public release; distribution is unlimited.

\author{
AFFTC-PA-10517
}


This Technical Information Memorandum, "Limited Investigation of Auxiliary Tasking during Boundary Avoidance Tracking" was prepared and submitted under Job Order Number MT080500 by the Commandant, USAF Test Pilot School, Edwards AFB, California, 93524-6485.

Prepared by:
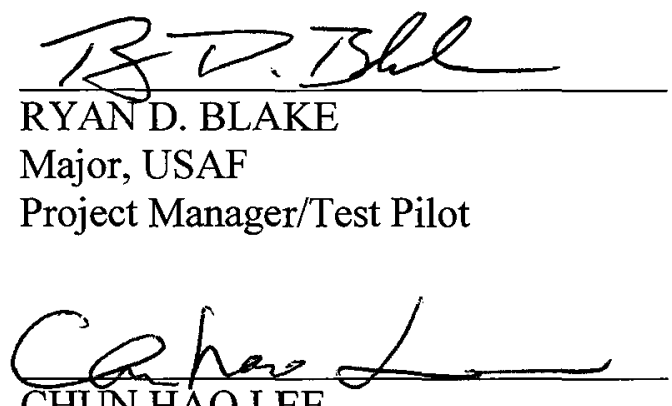

CHUN HAO LEE

Major, TAF

Test Pilot

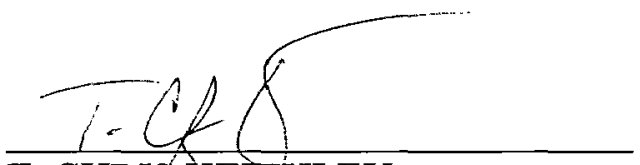

T. CHRIS KEIT/HLEY

Captain, USAF

Test Pilot

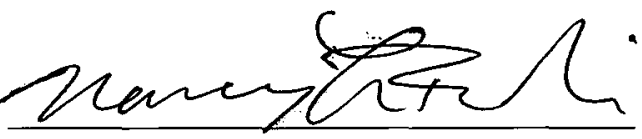

NANCY L. BACCHESCHI

Captain, USAF

Flight Test Engineer

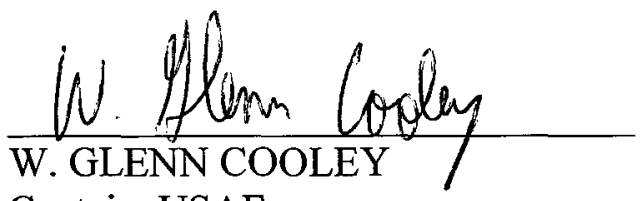

Captain, USAF

Flight Test Engineer
Reviewed by:
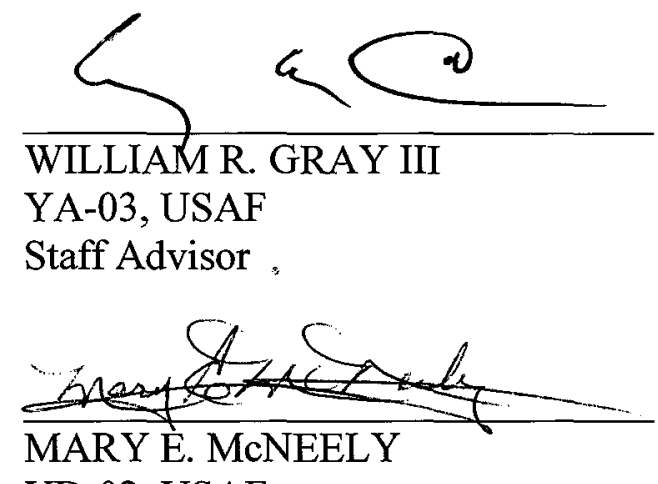

YD-02, USAF

Master Instructor, Test Management

Branch

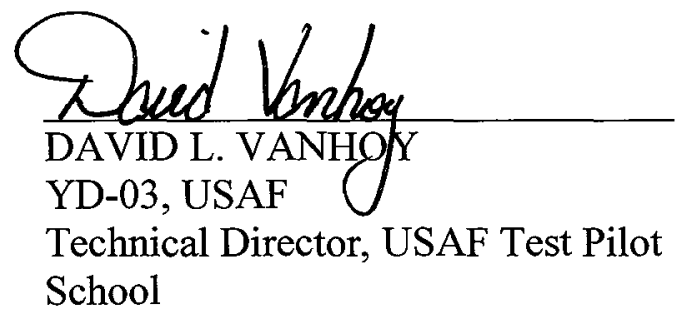

This memorandum

has been approved for publication:

Dery Mechuld twallex JUN 12010
TERKY M. LUALLEN
Colonel, USAF
Commandant, USAF Test Pilot School




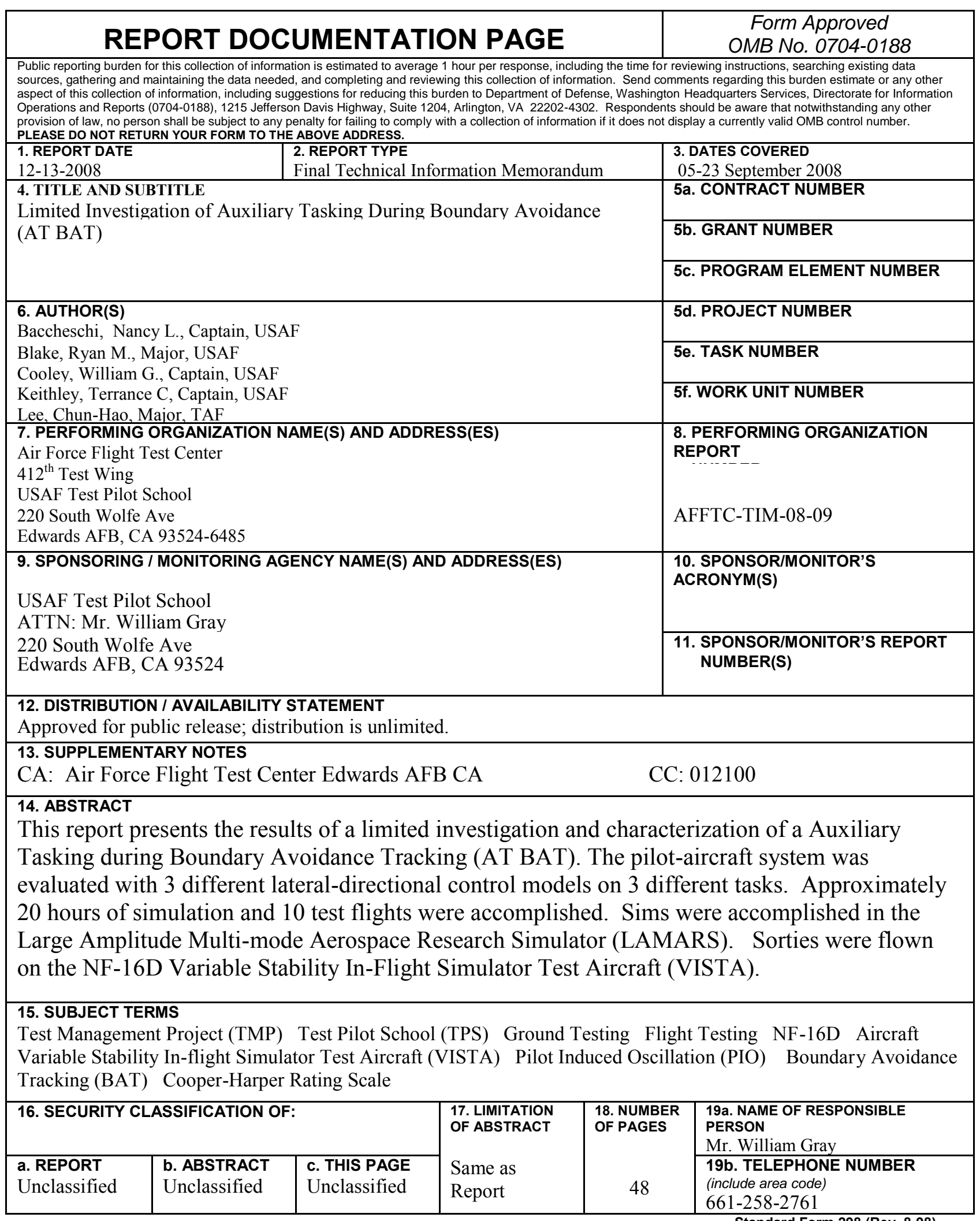


This page intentionally left blank. 


\section{EXECUTIVE SUMMARY}

This test report presents the results for the Auxiliary Tasking during Boundary Avoidance Tracking (AT BAT) Test Management Project (TMP). The AT BAT test team from the USAF Test Pilot School (TPS) at Edwards AFB, CA performed testing to research pilot behavior in the presence of boundaries.

The AT BAT TMP was conducted at the request of USAF TPS in collaboration with AFIT. The Commandant of USAF TPS directed this program. All testing was accomplished under TPS Job Order Number MT080500. Two calibration sorties and ten data sorties were flown on the Variable-stability In-flight Simulator Test Aircraft (VISTA) NF-16D aircraft, between 5 September, 2008, and 23 September, 2008, totaling 16.5 flight hours. Additionally, approximately 20 hours of simulation time were accomplished in the Large Amplitude Multi-Mode Aerospace Research Simulator (LAMARS) motion-based simulator at the Air Force Research Laboratory at Wright-Patterson AFB, OH.

This testing investigated the effects of imposing boundaries on a dynamic point tracking task in flight. Data collected during the flights in the LAMARS simulator and the VISTA, both programmed to simulate three aircraft with different handling qualities, were compared with the theoretical BAT model in order to determine the overall theory validity. In the tests, the pilot followed a point tracking task profile (a moving roll-angle target) displayed in the Heads-Up Display (HUD). The point tracking task profile also had boundaries, or limits, displayed in the HUD. As the tracking task progressed, the boundaries shrank in discrete increments. The boundary avoidance theory stated that as the boundaries encroached on the tracking task, the pilot would transition to a period of boundary awareness, where the shrinking boundaries would alter task performance. Eventually, as the boundaries got even closer, the pilot would transition to tracking the boundaries and abandon the point-tracking task entirely.

Previous BAT flight test research had focused on longitudinal maneuvering. This test program instead used lateral-directional maneuvering in the form of a roll-angle tracking task. In addition, this program implemented a secondary task (displayed in the HUD and tracked with a button on the throttle) as an objective measure of pilot workload.

The data from this test showed that pilots do react to boundaries. The average stick force and stick actuation rate tended to increase as the boundaries got closer, even though the underlying point tracking task did not change. In addition, the roll error (as a percentage of boundary size) increased dramatically at the lowest boundary size, suggesting that the point tracking task had been abandoned entirely. The theoretical BAT model was compared to pilot behavior during the tests, and was shown to match pilot inputs relatively well during periods of suspected boundary avoidance. Finally, the smallest achievable boundary size and the secondary task performance in a given aircraft were shown to correlate with the Cooper-Harper ratings given to that aircraft. 
This page intentionally left blank. 


\section{TABLE OF CONTENTS}

Page No.

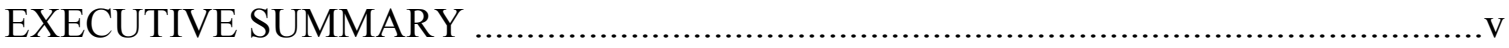

LIST OF ILLUSTRATIONS ................................................................................. vii

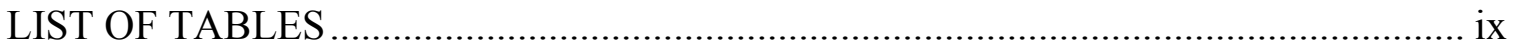

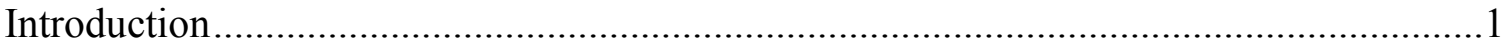

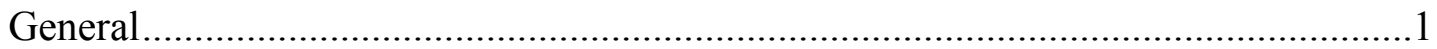

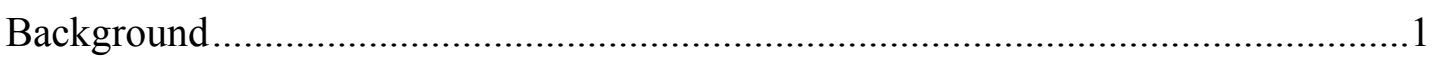

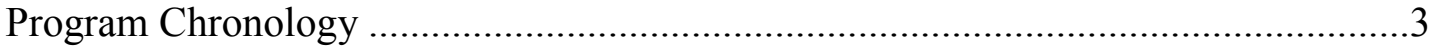

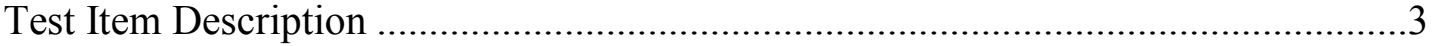

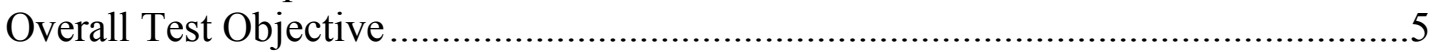

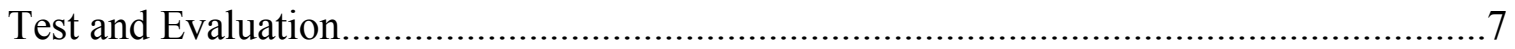

Aircraft Model Development.................................................................................

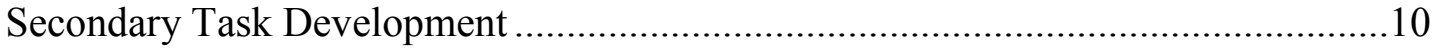

Data Analysis: Pilot Behavior in the Presence of Boundaries......................................12

Data Analysis: Cooper-Harper Ratings .................................................................2

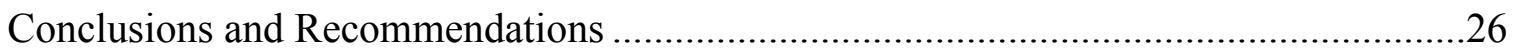

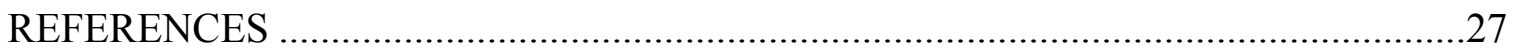

APPENDIX A - AIRCRAFT MODEL DESCRIPTIONS ......................................... A-1

APPENDIX B - COMPLETED TEST POINT MATRIX ……………………………....

APPENDIX C - COOPER-HARPER RATING SCALE ...............................................

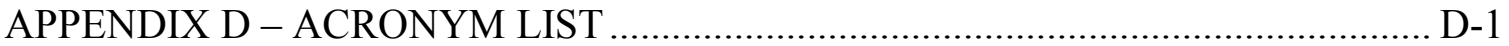




\section{LIST OF ILLUSTRATIONS}

Figure 1: Sample AT BAT HUD Symbology........................................................... 4

Figure 2: Cooper-Harper Task HUD Symbology ................................................... 7

Figure 3: BAT Task HUD Symbology .................................................................. 8

Figure 4: BAT Plus Secondary Task HUD Symbology ............................................ 9

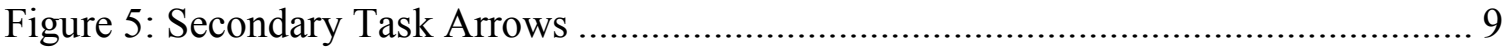

Figure 6: Pilot Crosscheck Ability (Eye-Tracker Data), Typical ................................. 11

Figure 7: Pilot Crosscheck Ability Compared with Secondary Task Error, Typical........ 12

Figure 8: Time History of Stick Force Input, Typical ............................................. 13

Figure 9: Average Duty Cycle and Aggressiveness (Average of All Runs).................... 14

Figure 10: Average Roll Error (Average of All Runs) ............................................... 15

Figure 11: Normalized Roll Error (Average of All Runs) ....................................... 15

Figure 12: LAMARS Pilot Input and BAT Predicted Input Time History ..................... 17

Figure 13: VISTA Pilot Input and BAT Predicted Input Time History ......................... 18

Figure 14: BAT Model Parameter Values ......................................................................... 20

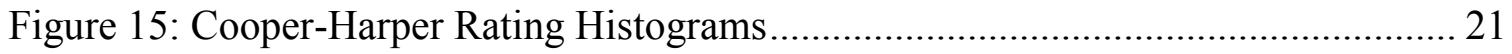

Figure 16: Cooper-Harper Ratings and Average BAT Task Run Time ......................... 22

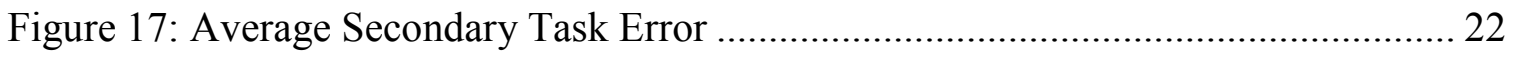

Figure 18: BAT Performance and Workload Correlated to Handling Qualities Levels... 25

Figure A-1: Roll Rate Response to Aileron Step, LAMARS .................................... A-2

Figure A-2: Long Term Roll Rate Response to Aileron Step, LAMARS .................... A-3

Figure A-3: Predicted Roll Rate Response to Aileron Step, VISTA ............................ A-5

Figure A-4: Flight Test Roll Rate Response to Aileron Step, VISTA.......................... A-6 


\section{LIST OF TABLES}

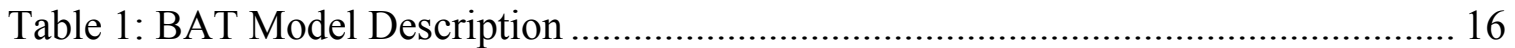

Table 2: BAT Prediction Values................................................................................... 18

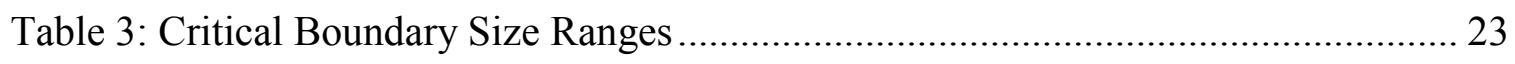

Table 4: Completed Test Point Matrix........................................................................ B-1 
This page intentionally left blank. 


\section{Introduction}

\section{General}

This Test Management Project (TMP) explored the Boundary Avoidance Tracking (BAT) concept. The first objective of the Auxiliary Tasking during BAT (AT BAT) program was to observe pilot behavior during a tracking task and secondary task in the presence of boundaries, and compare this behavior to the behavior predicted by the BAT theory. A second objective was to search for a correlation between the subjective pilot opinion ratings of the aircraft from a Cooper-Harper task and the objectivelymeasured performance and workload of a separate task in the roll axis.

The AT BAT TMP was conducted at the request of the Air Force Test Pilot School (TPS) in collaboration with the Air Force Institute of Technology (AFIT), Wright Patterson AFB, OH. The Commandant of USAF TPS directed the program. All testing was accomplished under TPS Job Order Number (JON) MT080500. It consisted of 12 test missions totaling 16.5 flight hours from 05 September, 2008, to 23 September, 2008, using the NF-16D Variable-stability In-flight Simulator Test Aircraft (VISTA).

\section{Background}

Previous pilot models, dating back as early as World War II, made the assumption that the pilot produces control actions based on observed error between a desired state and an actual state, be it regulation (e.g. keep the wings level) or tracking (e.g. climb/descend/turn to the appropriate altitude/heading). This type of control will subsequently be labeled point tracking.

In 2004, Mr. William Gray at the US Air Force Test Pilot School introduced the concept of Boundary Avoidance Tracking, challenging the assumption that a pilot only works to maintain certain conditions. He theorized that a pilot often acts to avoid a specific condition, or a boundary. An analogy to illustrate the difference involves a bicyclist maintaining a track along a line, such as a painted line on a road. Most people would have no trouble maintaining such a track. However, superimpose this white line across the Grand Canyon and the task becomes significantly more difficult. Most people would be well aware of the boundaries of their task, since crossing them would have fatal ramifications. Mr. Gray's research indicated that in such a scenario, the subject has lost awareness of the centerline altogether, using control solely to avoid the boundaries.

The next step in the development of this model was to attempt to match the model to experimental data. To do so, subjects flew a joystick-based desktop simulator in which the object was to follow a tracking bar moving up and down on the screen, while remaining within decreasing boundaries on either side of the tracking bar. Subjects were instructed to treat the boundaries as deadly; the task in fact stopped when a boundary was exceeded. During the simulations, the operators had little problem following the tracking bar when the boundaries were far apart, but had increasing difficulty tracking the bar as 
the boundaries closed in, often resulting in a pilot-in-the-loop oscillation (PIO) leading to boundary excursion. The data were analyzed during segments of suspected BAT activity (especially during the PIOs), and the BAT model predicted pilot inputs relatively well (reference 1).

The model was then explored during flight test. In 2005, TPS conducted flight tests in project HAVE BAT with T-38s to observe the BAT phenomenon in flight, refining the model's ability to predict pilot inputs (reference 2). The test aircraft was required to fly close formation with a target aircraft maneuvering longitudinally while maintaining position within certain visual references. An aircraft-mounted camera was placed in the test aircraft to record these visual references for use in data analysis. The data proved very difficult to extract from the video and severely limited BAT model characterization. However, the tests were successful in demonstrating BAT in flight and in suggesting improvements for future flight tests.

In 2006, TPS conducted another series of flight tests in project BAT DART, this time using the NF-16D VISTA (reference 3). The VISTA solved the problems inherent in the T-38 camera system by displaying a tracking task and boundaries in the Heads-Up Display (HUD), thereby eliminating the need for a target aircraft and video cameras. Data collection was instead accomplished with on-board software and data recording devices. The use of VISTA also permitted the simulation of four different aircraft-response models in one aircraft during the same flight. The primary focus of BAT DART was correlating pilot opinion ratings of the different simulated aircraft with tracking performance in the presence of boundaries. The tests were successful in demonstrating a qualitative relationship between pilot opinion ratings and both the length of time before the boundaries were exceeded and the average tracking error.

Previous tests involved only longitudinal maneuvering. The tracking tasks involved in these tests subjected the test pilots to large changes in normal acceleration (g), including large negative load factors. These changes in $\mathrm{g}$ were thought to influence pilot behavior, and perhaps be the source of an additional (and unwanted) boundary. This test program used a roll-angle task in order to eliminate this additional boundary and possibly provide "cleaner" data.

The previous attempt, in the BAT DART TMP (reference 3), to correlate BAT performance and Cooper-Harper ratings used only task performance in the attempted correlation. However, Cooper-Harper ratings require assessment of both performance and workload. Using a secondary, non-control task as a quantitative measure of pilot workload (i.e. pilot spare capacity) was used to solve this problem. This test added an additional, secondary task to the roll tracking task. The task involved actuating a switch in response to HUD symbology that changed in an unpredictable manner. In addition, eye-tracking equipment (available for use during simulator tests) provided information about pilot attention and workload during the profiles.

The tests were divided into three profiles. The first profile required tracking a roll angle for two minutes with defined performance criteria. The purpose of this profile was 
to assign a Cooper-Harper Rating to a given aircraft model. The second task required tracking a roll angle in the presence of boundaries. The purpose of this profile was to record pilot behavior during a tracking task in the presence of boundaries, and to provide a baseline for comparison with the secondary task profile. No Cooper-Harper rating was assigned at the end of this task. The last profile required tracking a roll angle in the presence of boundaries, with the additional task as described above. The purpose of this profile was to record pilot behavior during a tracking task in the presence of boundaries and a secondary task. The performance of the secondary task provided an indication of pilot workload (i.e. "spare capacity" during the primary roll-angle tracking task). No Cooper-Harper rating was assigned at the end of this task.

Fixed-base simulation was accomplished as part of an in-progress AFIT Master's thesis at AFRL. These simulations were structured very closely to the tests performed during this test program. The tests used a roll-angle tracking task displayed in the HUD, and a secondary task consisting of four arrows which illuminated one-at-a-time in a Heads Down Display. The primary task performance (measured as minimum boundary size achievable) was shown to worsen as the aircraft handling qualities worsened. The secondary task performance was shown to remain approximately constant until a certain boundary size (dependent on the aircraft handling qualities) was reached, at which point performance decreased. This point was labeled the "boundary awareness point". A combination of primary and secondary performance was shown to correlate well with Cooper-Harper ratings.

Motion-based simulation was also accomplished by the student test team at the full-motion Large Amplitude Multi-Mode Aerospace Research Simulator (LAMARS) simulator at AFRL. A draft of the flight test plan was implemented in the simulator, with improvements incorporated into the flight test plan.

\section{Program Chronology}

A joint Technical Review Board/Safety Review Board (TRB/SRB) was conducted on 25 Aug 08. The TRB was chaired by Mr. Gary Aldrich, USAF TPS/EDT. The SRB was chaired by Mr. Rob Warner of AFFTC/SET.

The test project consisted of three full days of simulator studies at the LAMARS, a ground checkout, and twelve NF-16D VISTA sorties totaling 16.5 flight hours within the R-2508 complex at Edwards Air Force Base, California. The simulator studies occurred on 16-18 July 2008. Ground tests occurred on 03 and 04 September 2008. Flight testing occurred from 05 to 23 September 2008.

\section{Test Item Description}

The NF-16D VISTA was a modified Block 30 F-16D, based on the Israeli Air Force's airframe design. The avionics were Block 40 configuration, and a custom Digital Flight Control Computer (DFLCC) was installed. The Variable Stability System (VSS) was a five-degree-of-freedom system used to simulate various types of aircraft handling 
qualities by changing the static and dynamic flight characteristics. The front seat was the simulation cockpit and the rear seat had the primary flight controls.

With the VSS engaged, the Evaluation Pilot (EP) in the Front Cockpit (FCP) could control the aircraft with either a centerstick or sidestick, using the programmed control laws. This project used the sidestick controls. As a safety feature, the Safety Pilot (SP) in the Rear Cockpit (RCP) maintained pilot-in-command features. All displays were programmed to the user's needs.

Specific modifications to the NF-16D aircraft for the AT BAT project include:

1) Custom VSS programming to simulate 3 different aircraft's lateral/directional control laws.

2) Custom display programming to input a target and boundary profile into the HUD, as well as a secondary task in the lower left-hand corner, as shown in figure 1.

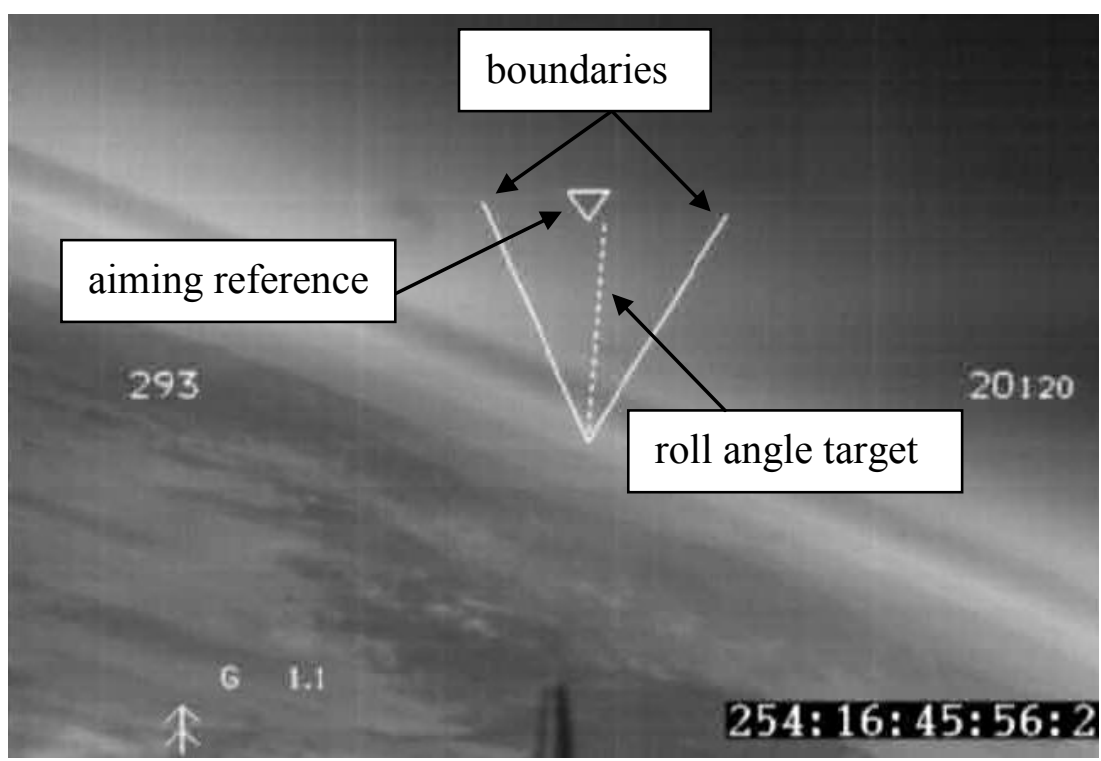

Figure 1: Sample AT BAT HUD Symbology

The VISTA used HUD and VSS video tapes to record data during flight.

Calspan, which was in charge of VISTA operation, designed three aircraft models for this test. The intent was to make models with varying levels of degraded lateral-directional handling qualities. See the aircraft model development section and appendix A for specific information about the models and the development procedure. 


\section{Overall Test Objective}

The primary objective of the AT BAT program was to observe pilot behavior during a tracking task and secondary task in the presence of boundaries and to compare this behavior to the behavior predicted by the BAT theory. A second objective was to investigate a correlation between the subjective pilot opinion ratings of the aircraft from a Cooper-Harper task and the objectively-measured performance and workload of a separate task in the roll axis. All objectives were met. 
This page intentionally left blank. 


\section{Test and Evaluation}

Flight test was accomplished from 05 to 23 September, 2008. Twelve sorties were flown for 16.5 flight hours in NF-16D 86-00048.

The first two flights were used to ensure the aircraft models provided by Calspan were controllable and provided a sufficient handling qualities spread, in terms of their ability to satisfactorily accomplish the roll angle tracking task. Each configuration was tested along with programmed test inputs applied by the VSS.

Data for the AT BAT TMP were collected on the remaining ten flights. Each member of the test team flew twice. Flight test engineer data was included in the analysis and showed no bias to lack of experience. The profile order was randomized between flights and within flights to preclude the test subject anticipating and/or learning what the next configuration was.

Prior to each test, the aircraft was trimmed in straight and level, unaccelerated flight at the prescribed flight condition of $300 \mathrm{KCAS}$ and 20,000 ft PA. The evaluation pilot initiated the profile using a button on the control stick.

Three types of profiles were flown on each sortie.

- For the Cooper-Harper task (figure 2), the roll-angle target was displayed in the HUD. The pilot attempted to keep the target aligned with a caret at the top of the HUD. A set of lines fixed at \pm 5 degrees from the target defined the boundaries of desired performance, and a set of lines fixed at \pm 7.5 degrees from the target defined the boundaries of adequate performance. The pilot assigned a Cooper-Harper rating (see appendix C) based on performance and workload during the profile.

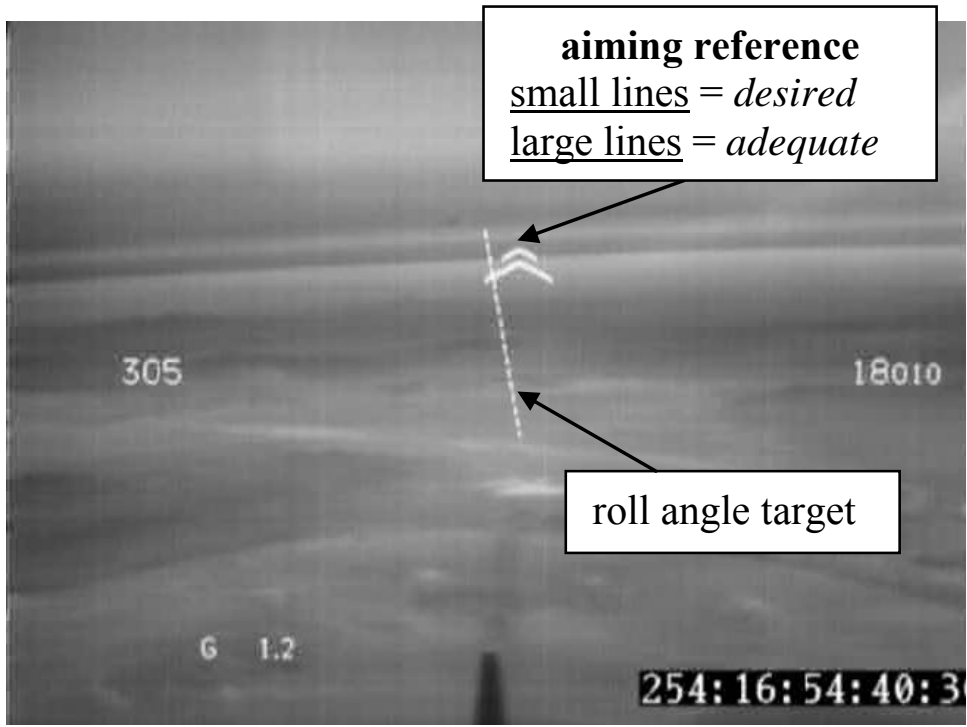

Figure 2: Cooper-Harper Task HUD Symbology 
- For the BAT task (figure 3), the roll-angle target was displayed as in the previous profile. The 5 degree and 7.5 degree lines from the previous profile were replaced with a single set of boundaries starting at \pm 30 degrees. The boundaries remained constant for 30 seconds, and shrank by approximately 6 degrees at the end of the 30 second period. The boundaries continued to shrink by approximately 6 degrees every 30 seconds until the end of the profile. (Note that the boundary profile requested by the test team began at \pm 40 degrees and shrank by 20 percent every 30 seconds. Due to programming anomalies, the actual boundary profile implemented in the VISTA is as described above.) The pilot treated the boundaries as life-threatening, and maneuvered as required to prevent exceeding them. The profile stopped automatically when the fixed caret at the top of the HUD exceeded the boundaries for 0.5 seconds. The purpose of the 0.5 second delay was to gather data for pilot behavior outside given boundaries.

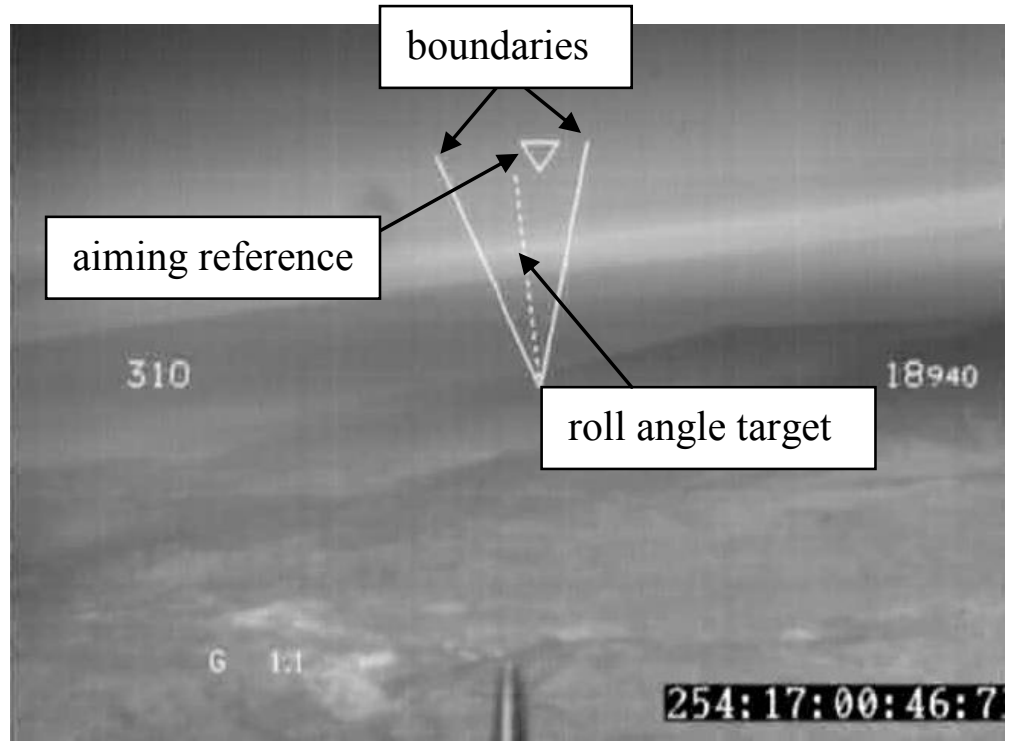

Figure 3: BAT Task HUD Symbology

- For the BAT plus secondary task (figure 4), all procedures were the same as the previous profile, except that a secondary task was displayed in the HUD. A set of four arrows illuminated one at a time, and the pilot deflected the radar cursor switch on the throttle in response to the illuminated arrow. The arrows consisted of a single-headed arrow pointing up, a double-headed arrow pointing up, a single-headed arrow pointing down, and a double-headed arrow pointing down (see figure 5). The cursor control was deflected up once for the single-headed arrow pointing up, and it was deflected twice for the double-headed arrow pointing up. The switch was deflected down once for the single-headed arrow pointing down, and it was deflected twice for the double-headed arrow pointing down. As before, the pilot treated the roll-angle tracking task as the primary task, and treated the boundaries as the 
primary threat. The secondary task was neglected as required to prevent exceeding the boundaries.

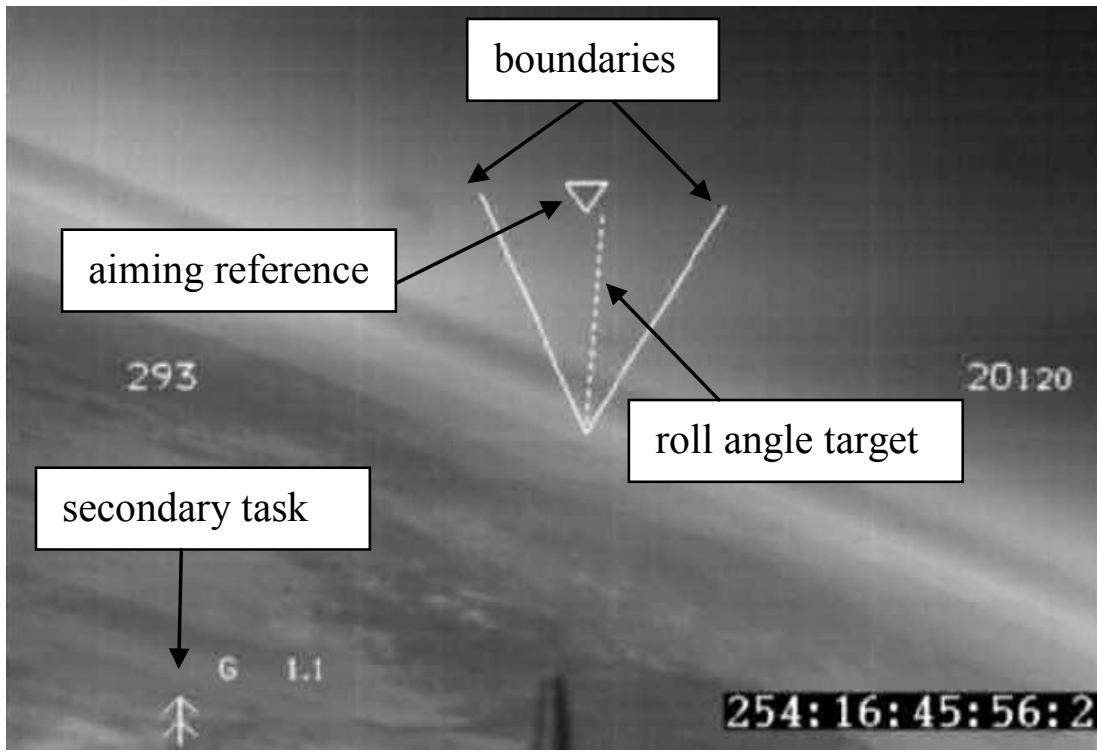

Figure 4: BAT Plus Secondary Task HUD Symbology

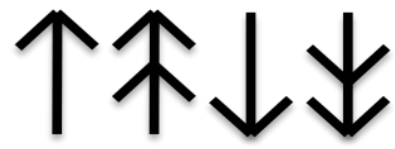

Figure 5: Secondary Task Arrows

The first profile of each sortie was a 30 second Cooper-Harper roll-tracking task using a Level 1 configuration flown as a warm-up to acquaint the subject with the symbology. Subsequent profiles were selected using a randomized test matrix (see appendix B). Within the matrix were a 2-minute Cooper-Harper task, a BAT task, and a BAT task with a secondary task for each of the aircraft models. Thus, each sortie included ten total profiles, nine of which were data profiles.

\section{Aircraft Model Development}

Calspan, which was in charge of the test aircraft operation, designed three aircraft models for this test. The intent was to make Class IV, Phase A aircraft models with Level 1, Level 2, and Level 3 lateral-directional handling qualities, respectively. The models were developed using roll mode and Dutch roll parameters from MIL-STD1797B for a Category IV aircraft in flight phase A (reference 4). For the proposed Level 1 aircraft, the roll mode time constant and Dutch roll frequency and damping were chosen from the suggested Level 1 values in the MIL-STD. Likewise, the proposed Level 2 aircraft had a roll mode time constant and Dutch roll frequency and damping that 
each were predicted to result in Level 2 handling qualities. The proposed Level 3 aircraft was designed in the same way. See appendix A for specific model descriptions.

The proposed models were tested in the Large Amplitude Multi-Mode Aerospace Research Simulator (LAMARS) simulator, and did not have the desired spread in handling qualities. The three models had noticeably different handling qualities, but were each (in their own way) approximately Level 2 aircraft. The data from the LAMARS simulator were useful for this test program in that it showed the models should be modified to provide a larger spread in handling qualities.

Additionally, the Cooper-Harper task was adjusted in an attempt to allow for a spread in performance and workload that would result in Levels 1,2 and 3. This was done by adjusting the percentages for desired and adequate criteria until the level of difficulty and workload for the task was appropriate to allow for a spread in CooperHarper ratings.

As a result of the LAMARS simulations, Calspan modified the aircraft models to attempt a larger spread in handling qualities. These new models were implemented in the VISTA. Due to uncertainty in the VISTA aerodynamic model, the handling qualities during the calibrations flights were dramatically different than predicted. The first calibration sortie showed that the proposed Level 2 and Level 3 aircraft were actually uncontrollable. Calspan made changes to the models, and the second calibration sortie demonstrated some improvement, but the proposed Level 3 aircraft was still uncontrollable.

After the calibration sorties, the team decided that too many variables were changing among the models, and that a model with parameters that were separately predicted to be Level 3 would in combination lead to an uncontrollable aircraft. As a result, the final models were all given roughly the same Dutch roll characteristics, and the roll mode time constant was used to change the models. The achieved handling qualities of the models are described below in the Data Analysis. The three models were given the names "Aircraft X", Aircraft Y", and "Aircraft Z." See appendix A for specific model descriptions.

\section{Secondary Task Development}

The test project team designed two secondary task profiles for this test. It was expected that the performance in the secondary task would decrease as boundaries shrink, to the point where the pilot would abandon altogether the secondary task. The task included four arrows illuminated on the lower-left corner of the HUD one at a time and changed every 2 to 4 seconds.

In the LAMARS simulator sessions, the team also added an eye-tracker system to record pilots' eye focal point when flying with secondary tasks. The eye-tracker was not cleared for flight test and not used in the VISTA sorties. The data included a series of 24 frames-per-second, MPEG-4 digital video in 752x480 pixel resolution. The eye-tracker 
used a Charge-Coupled Device (CCD) to track the center of pilot's right pupil and another CCD to record in the direction the pilot's head was pointed. The equipment then superimposed a red cross (as the center of pupil) on the front view video data. Eye-tracker performance was limited by its lens field-of-view (roughly \pm 15 degrees), CCD sensitivity, and bore-sight alignment. Additionally, when the pilot blinked, the eye-tracker would lose track and the cursor would jump back to the origin. Even with these limitations, the team still gathered representative data to show aircraft with varying levels of handling qualities affected the crosscheck performance of the pilot.

Figure 6 shows a sample of a single pilot's secondary task crosscheck for each aircraft model. A crosscheck was defined as a movement of the pilot's eye from the primary task to the secondary task. The crosscheck frequency was defined as the number of crosschecks divided by the time between boundary changes (30 seconds). Decreasing crosscheck meant that the pilot was looking at the secondary task less often. As expected, the crosscheck ability of the pilot decreased as the boundaries decreased, and also decreased in proportion to decreasing handling qualities.

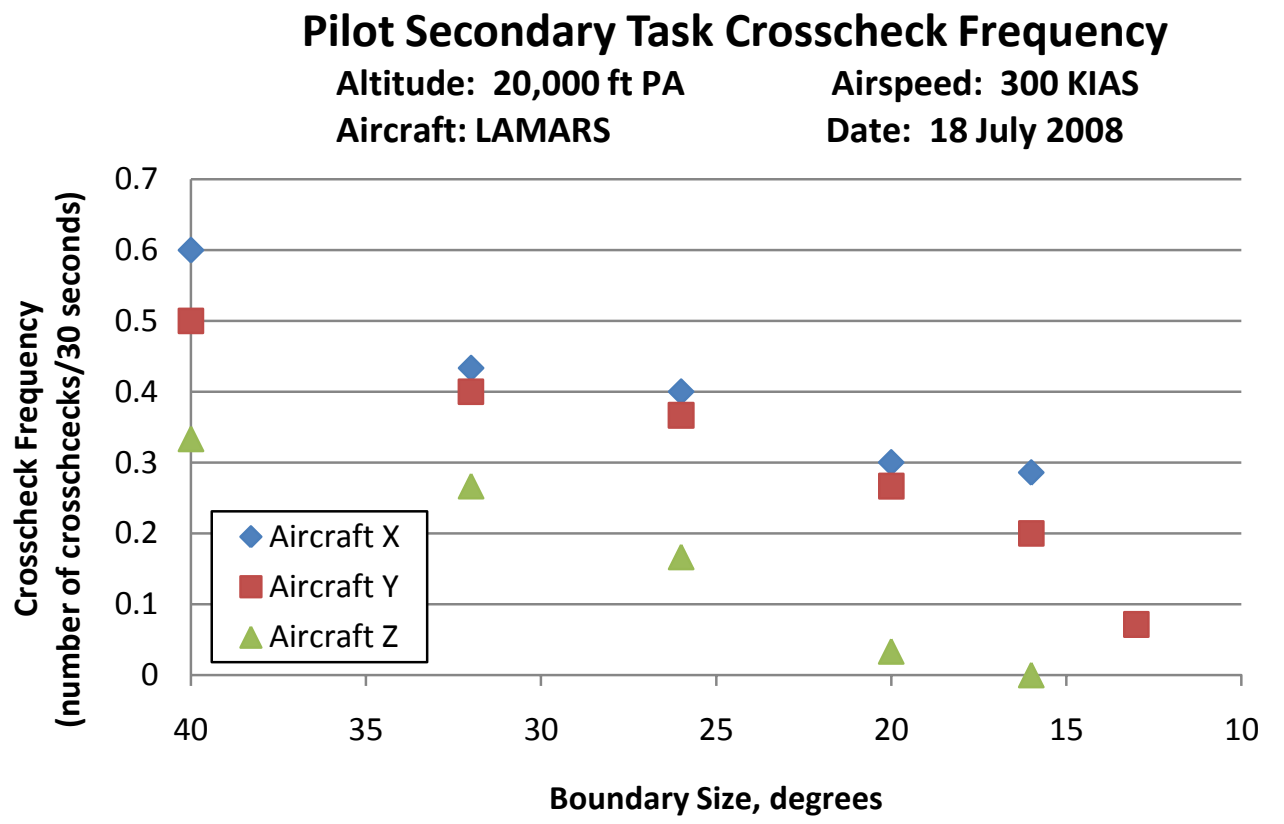

Figure 6: Pilot Crosscheck Ability (Eye-Tracker Data), Typical

The crosscheck frequency also helped validate the secondary task as a measure of pilot spare capacity. Figure 7 shows a typical pilot's crosscheck ability compared to his secondary task performance. The trends of the two sets of data followed each other closely, which suggested that the secondary task alone could be used in the VISTA as a measure of pilot spare capacity (since the eye-tracker was not cleared for flight test). 


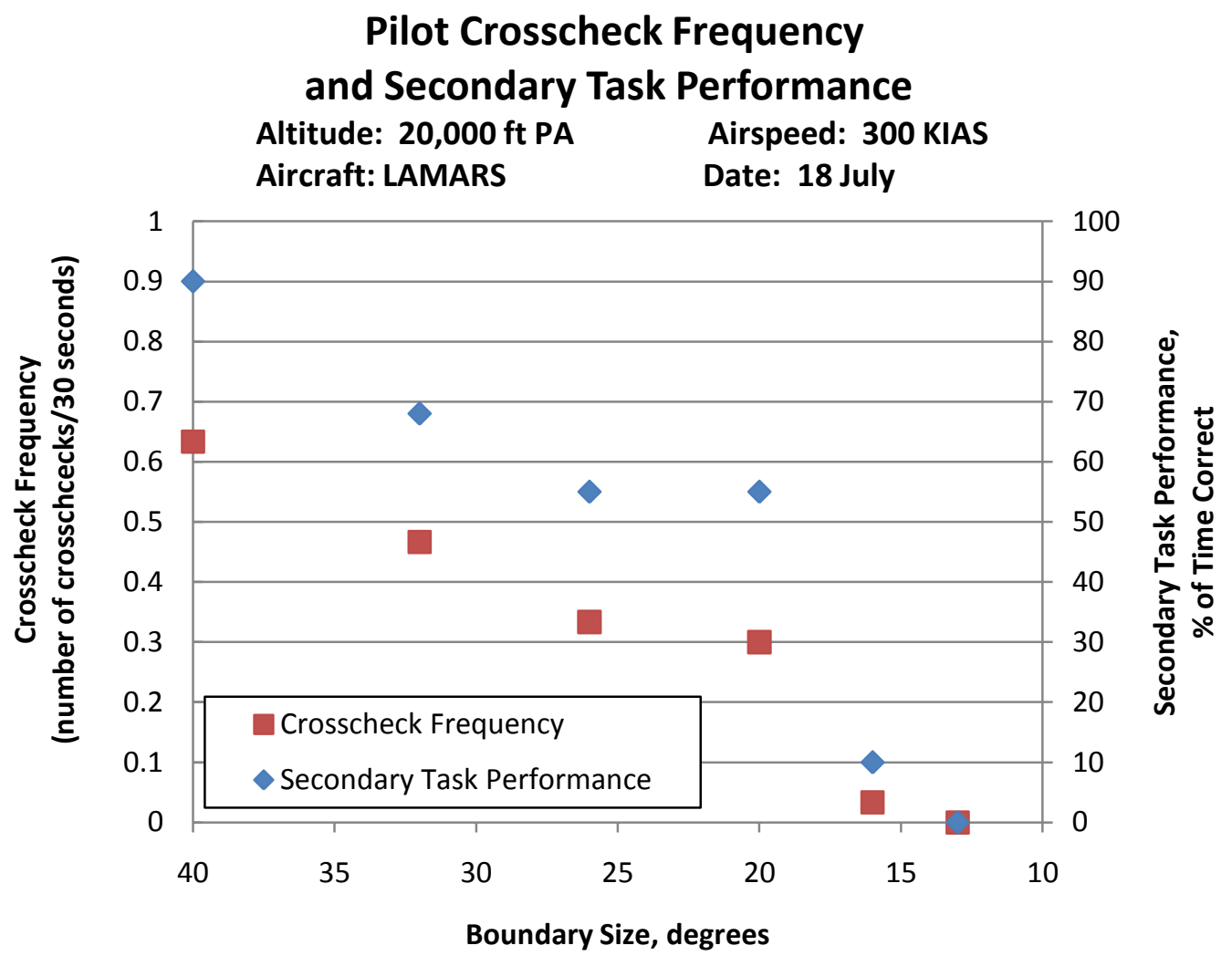

Figure 7: Pilot Crosscheck Ability Compared with Secondary Task Error, Typical

The Calspan software developer indicated that they could only program the secondary task switch for two directions in the VISTA, so the tasks were changed to accommodate this limitation (see the beginning of the test and evaluation section for final symbology). The test team agreed that this would not change the difficulty of the secondary tasks, and thus would not affect their use as measures of pilot workload.

\section{Data Analysis: Pilot Behavior in the Presence of Boundaries}

The first objective of the AT BAT project was to observe pilot behavior in the presence of boundaries to determine if the presence of those boundaries altered the pilot's behavior in any manner. An examination of the pilot's stick inputs as a function of time shows that the pilot does indeed change his behavior as a function of decreasing boundaries. For the BAT and BAT plus secondary tasks, the only part of the task that was changing with time was the boundary size. The roll task itself was constant throughout the time history. As illustrated in figure 8, the force the pilot input on the stick increased with time. This was typical of most of the pilots' force trends in both LAMARS and the VISTA. The only part of the task that was changing was the decreasing boundaries (indicated on the plot in the stairstep), indicating that the pilot used greater force on the stick as the boundaries became tighter. 


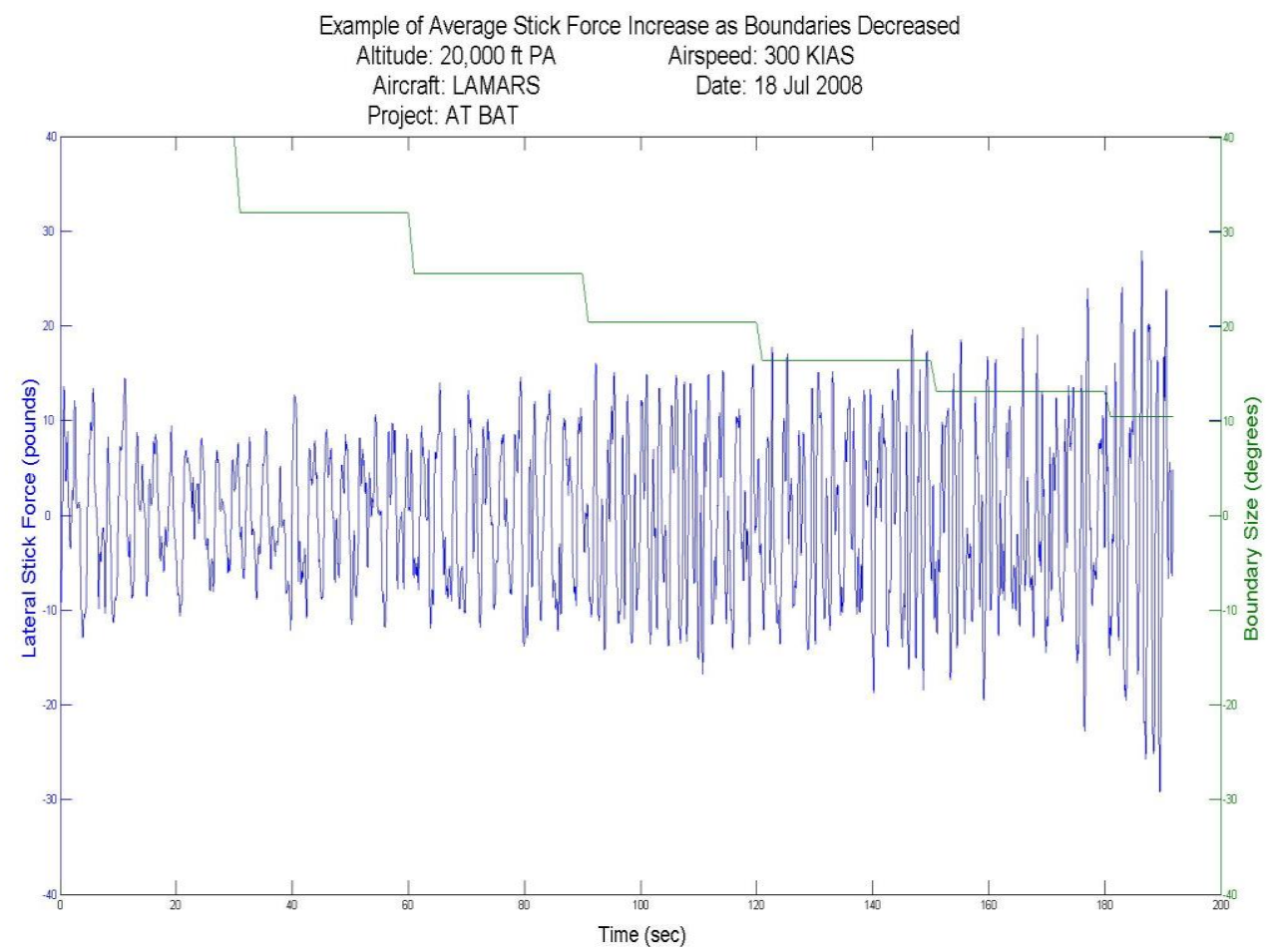

Figure 8: Time History of Stick Force Input, Typical

This changing pilot behavior was also analyzed through the concept of duty cycle and aggressiveness (reference 5). This concept was a way of looking at pilot "gain", by describing how often and how aggressively the inceptor was moving. Duty cycle was defined as the percentage of time that the inceptor was moving above a defined threshold (set at 5 pounds per second). Aggressiveness was defined as the average rate of inceptor movement. A high duty cycle and low aggressiveness, for example, would suggest that the pilot was constantly moving the inceptor, but at small rates.

Average duty cycle and aggressiveness were calculated for each aircraft at each boundary size, and are plotted in figure 9. The data show that for each aircraft, aggressiveness increased as the boundaries decrease. This mirrored the results shown in figure 8 above, and further confirmed the theory that boundaries change pilot behavior. The duty cycle also increased as boundaries decreased for Aircraft X and Y. However, duty cycle was relatively constant with boundary size for Aircraft Z. This was likely due to the poor handling qualities of Aircraft Z, requiring the pilot to "stay out of the loop" as much as possible in order to maintain appropriate control. Also note that the decrease in duty cycle and aggressiveness for Aircraft $Y$ in LAMARS at the 10.5 degree boundary size was due to the fact that only three pilots were able to operate to this boundary size, and their duty cycle and aggressiveness was, on average, lower than the other pilots. 

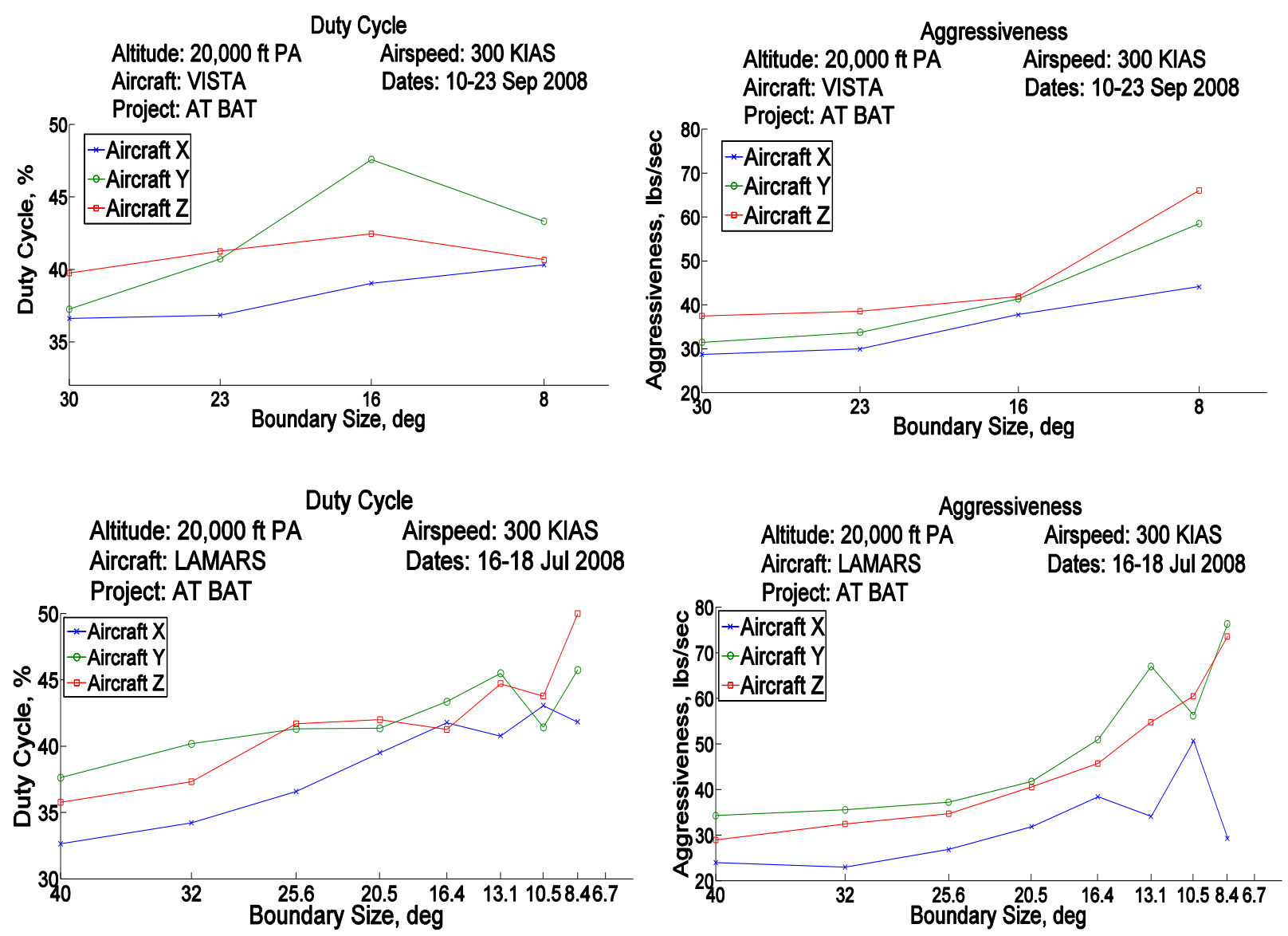

Figure 9: Average Duty Cycle and Aggressiveness (Average of All Runs)

In addition to affecting inceptor movement, boundaries also affected the pilot's tracking performance. With large boundaries, the required pilot performance was low. As the boundaries shrank, the required performance increased, and the tracking performance subsequently increased (i.e. the roll error decreased), even though the tracking task had not changed. Figure 10 shows the general trend of increasing performance as a function of decreasing boundary size. 

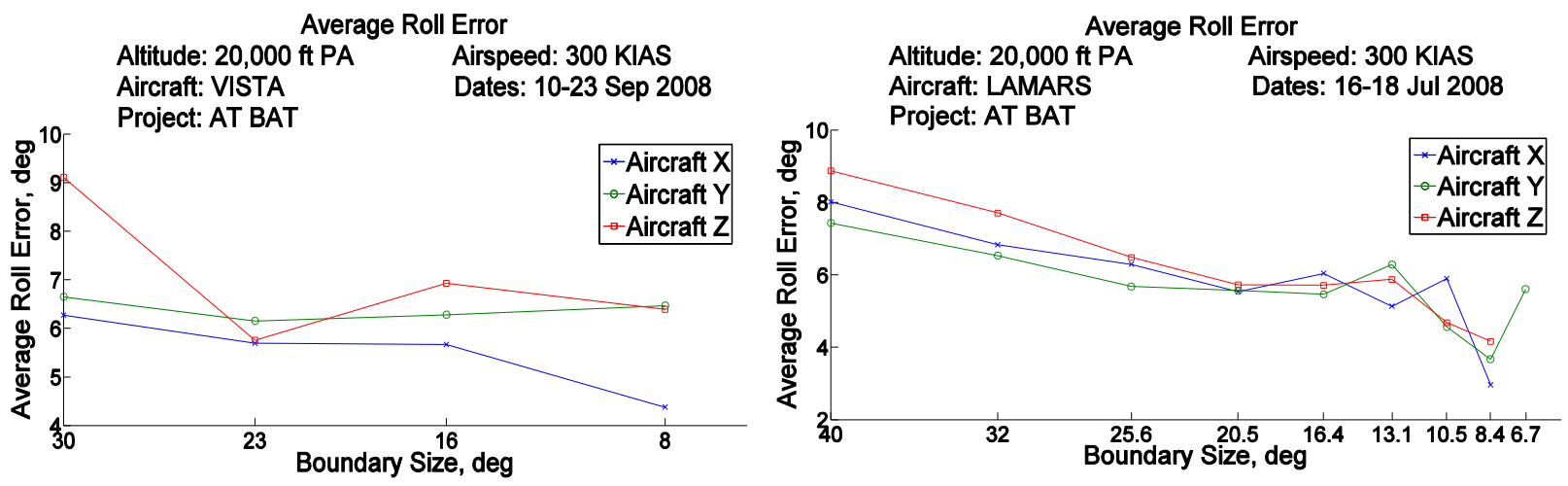

Figure 10: Average Roll Error (Average of All Runs)

Additional insight was gained by plotting the roll error as a percentage of boundary size. While the absolute error (figure 10) decreased as a function of boundary size, the normalized error (figure 11) remained roughly constant until the boundaries decreased to a given size, at which point the normalized error began to increase and performance decreased. From these data, it can be concluded that increasing performance requirements increased performance until the requirements were too stringent, at which point the increasing requirements actually decreased performance.
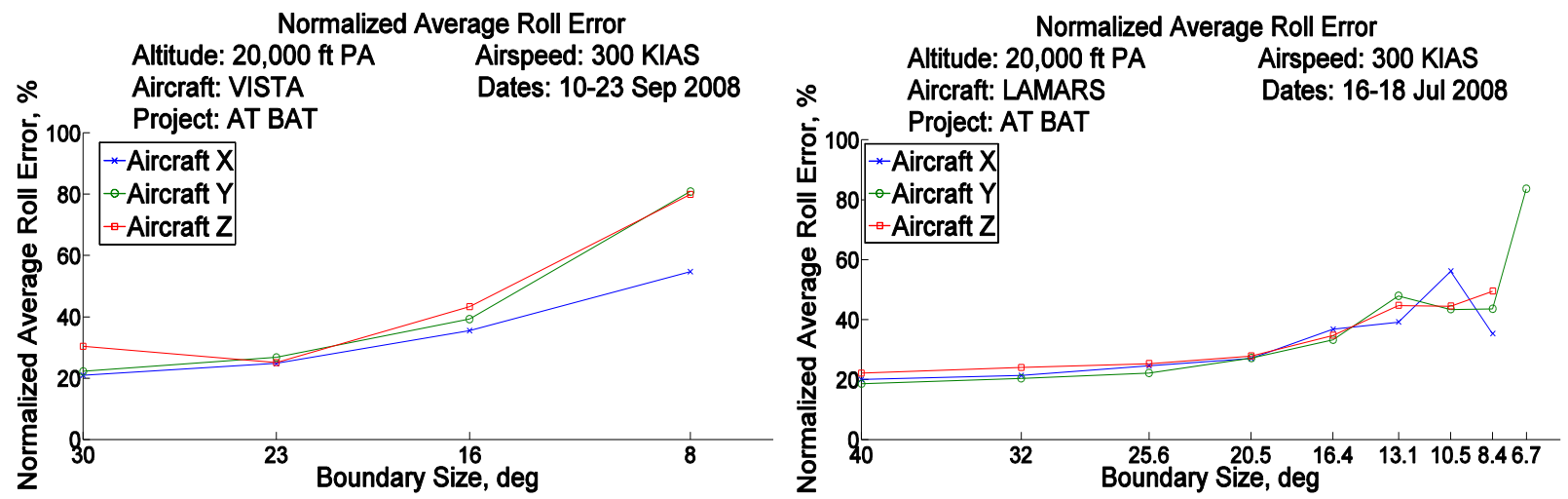

Figure 11: Normalized Roll Error (Average of All Runs) 
Another objective of the AT BAT project was to quantitatively model a pilot's behavior in the presence of boundaries. The proposed BAT pilot model was a switching model, in which a pilot engaged in point tracking (i.e. making inputs solely in response to tracking error) until boundaries became a factor (i.e. until the pilot felt that boundaries were likely to be exceeded). When boundaries became a factor, the pilot in the proposed model began tracking the boundaries by making inputs solely to avoid the boundaries (and neglecting the point tracking task). In this model, the pilot was described by table 1 (reference 1):

Table 1: BAT Model Description

\begin{tabular}{|l|l|c|}
\hline Situation & Boundary Awareness & Boundary Feedback \\
\hline $\begin{array}{l}\text { Displacement inside, } \\
\text { moving away from boundary }\end{array}$ & No threat & 0 \\
\hline $\begin{array}{l}\text { Displacement inside, } \\
\text { moving toward boundary }\end{array}$ & & \\
\hline$t_{b} \geq t_{\min }$ & No threat & 0 \\
\hline$t_{\max }<t_{b}<t_{\min }$ & $\begin{array}{l}\text { Feedback increases linearly as } \mathrm{b}_{\mathrm{b}} \\
\text { descreases }\end{array}$ & $\frac{t_{\min }-\left(t_{b}+\tau_{b}\right)}{t_{\min }-t_{\max }} K_{b m}$ \\
& $\begin{array}{c}\text { where } \\
t_{b}=x_{b}\left(d x_{b} / d t\right)^{-1}\end{array}$ \\
\hline$t_{b} \leq t_{\max }$ & Maximum threat & $K_{b m}$ \\
\hline Displacement outside boundary & Maximum threat & $K_{b m}$ \\
\hline
\end{tabular}

Where:

$t_{\min }$ : The minimum time to boundary at which the pilot first began reacting to the boundaries; a larger value of $t_{\min }$ implies that the pilot begins reacting to boundaries sooner

$\mathrm{t}_{\max }$ : The time at which the pilot input the maximum force to avoid a boundary; $a$ larger value of $\mathrm{t}_{\max }$ implies that the pilot applies maximum force sooner $t_{b}$ : The time to boundary based on the boundary size and the pilot's error and roll rate

$\tau_{\mathrm{b}}$ : Pilot time delay

$\mathrm{K}_{\mathrm{bm}}$ : The max stick force the pilot input

$\mathrm{x}_{\mathrm{b}}$ : Distance to boundary 
Equation 1 in the table described pilot inputs only during periods of boundary tracking. When boundaries were not a factor, the BAT equation predicted zero force. At this point, a point tracking model would need to be used to predict point tracking force. For this test, a time history of the pilot's stick force inputs was plotted against a BAT prediction of the inputs. The values were tuned until there was agreement between what the pilot actually did in the test and what the BAT model would have predicted. The tuning was an iterative process whereby the values for each of the variables described in table 1 were changed in small increments until some combination of all the values resulted in a predicted input that matched the actual pilot input. Only periods of suspected boundary avoidance (from pilot comments or observed behavior) were analyzed for this prediction. Example results from a LAMARS profile and a flight profile in the VISTA are shown in figures 12 and 13.

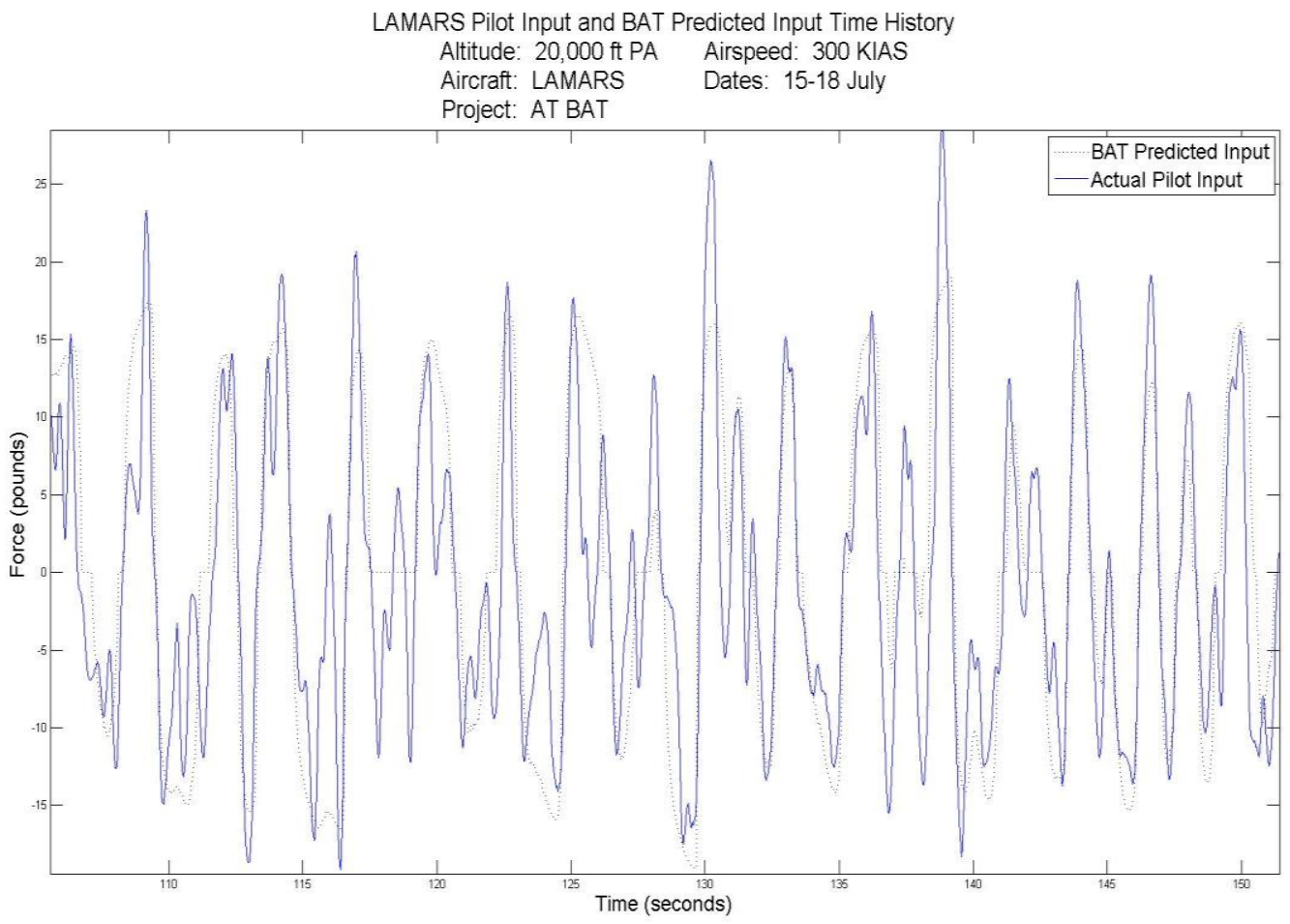

Figure 12: LAMARS Pilot Input and BAT Predicted Input Time History 


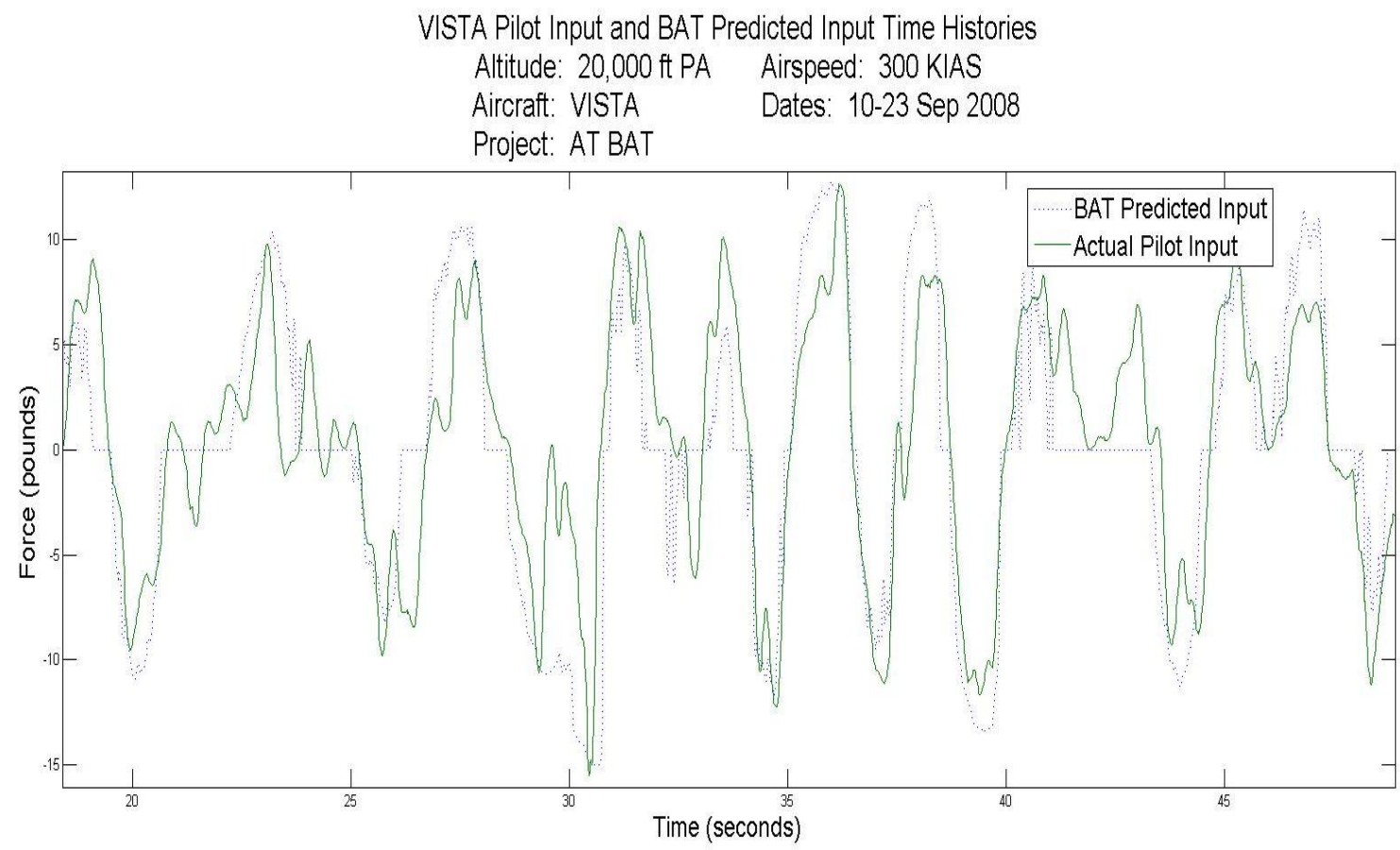

Figure 13: VISTA Pilot Input and BAT Predicted Input Time History

The model tuning was accomplished for every BAT and BAT plus secondary task profile flown in both LAMARS and the VISTA, for a total of 137 profiles. The ranges of values that resulted in good agreement between actual and predicted inputs are shown in table 2 .

Table 2: BAT Prediction Values

\begin{tabular}{|c|c|c|}
\hline & LAMARS & VISTA \\
\hline $\mathbf{t}_{\mathbf{m i n}}$ (seconds) & & \\
\hline $\min$ & 2 & 1.3 \\
\hline $\max$ & 5 & 5 \\
\hline $\mathbf{t}_{\mathbf{m a x}}(\mathbf{s e c o n d s})$ & & \\
\hline $\min$ & 0.06 & 0.06 \\
\hline $\max$ & 0.1 & 0.4 \\
\hline $\boldsymbol{\tau}_{\mathbf{b}}$ (seconds) & & \\
\hline $\min$ & 0.2 & 0.15 \\
\hline $\max$ & 0.25 & 0.25 \\
\hline $\mathbf{K}_{\mathbf{b m}}$ (pounds) & & \\
\hline $\min$ & 10 & 11 \\
\hline $\max$ & 28 & 19 \\
\hline
\end{tabular}

There are a few interesting items to note from the table and plots of the BAT predictions. First, there was generally better agreement between the prediction and actual inputs in the LAMARS data for nearly the entire time history, even when it could be argued the pilot was acting only to follow the roll task and was not close enough to a 
boundary to be exhibiting boundary avoidance behavior. In the VISTA comparisons, it was only possible to get acceptable agreement where the pilot was clearly exhibiting boundary avoidance behavior. This was defined as any point in the time history where the pilot exceeded or came close to exceeding a boundary and/or where he exhibited pilot-in-the-loop oscillations (PIOs). PIOs were determined as any point where the pilot-commanded roll angle oscillated around the roll task angle for more than two cycles. It was at these points that the BAT prediction parameters were tuned to match the actual pilot inputs. Given that the pilot had less to split his time and focus on in the simulator than the actual flight test, it is possible that the addition of the real-world variables introduced in the flight test sorties caused larger differences between the model and the actual outputs.

Figure 14 shows the range of values for each of the BAT model parameters for both the VISTA flights and the LAMARS data. Both sets of data included three different aircraft, trying to model three different levels of handling qualities. This resulted in six different aircraft models between the LAMARS and VISTA sorties.

Overall, the $t_{\min }$ values in the VISTA sorties were shifted lower than in the LAMARS data. This indicates that in the LAMARS simulations the pilots were reacting to boundaries sooner than in the VISTA sorties. It is possible that the pilot was experiencing more "seat-of-the-pants" motion in flight tests than in the simulator, and as a result was busy managing other tasks, such as maintaining altitude, thereby delaying any reactions to boundaries until he was closer to the boundaries. This "seat-of-the-pants" feeling would also explain why there was a larger spread and higher overall value for $t_{\max }$ in the VISTA data than in the LAMARS data. The higher $t_{\max }$ indicates that the pilot was inputting maximum force even when he was farther from the boundaries than in the LAMARS data. The true motion of the aircraft was a better indicator than even a motion-based simulator for such parameters as the rate he was approaching a boundary, which would explain why he was reacting with maximum force to a boundary sooner than in the LAMARS data.

Additionally, there was a greater range of pilot delay time $\left(\tau_{\mathrm{b}}\right)$ in the VISTA data. The motion of the aircraft was likely indicating to the pilot sooner that he needed to react, which would explain why in some cases the pilot delay was shorter than in LAMARS. There was a tighter grouping of max force $\left(\mathrm{K}_{\mathrm{bm}}\right)$ in the VISTA data as well. This again was likely due to a stronger motion cue in flight data than in the simulator, allowing the pilot to shape his inputs more precisely in the VISTA than in the LAMARS data. 

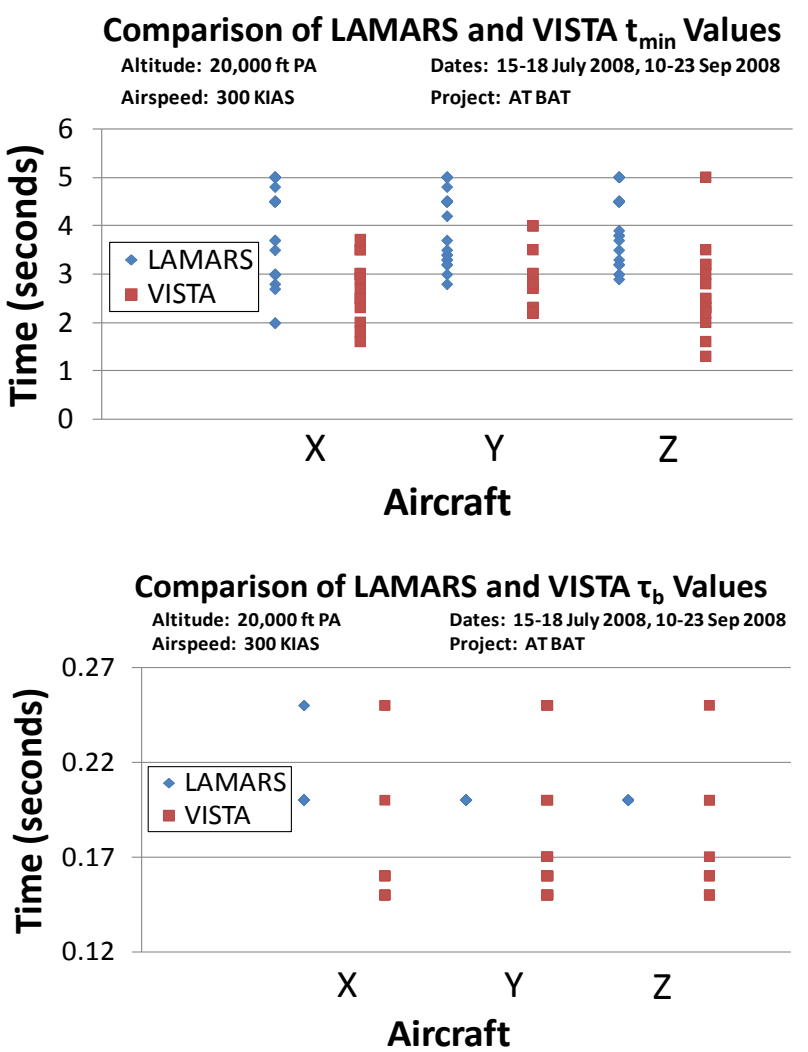
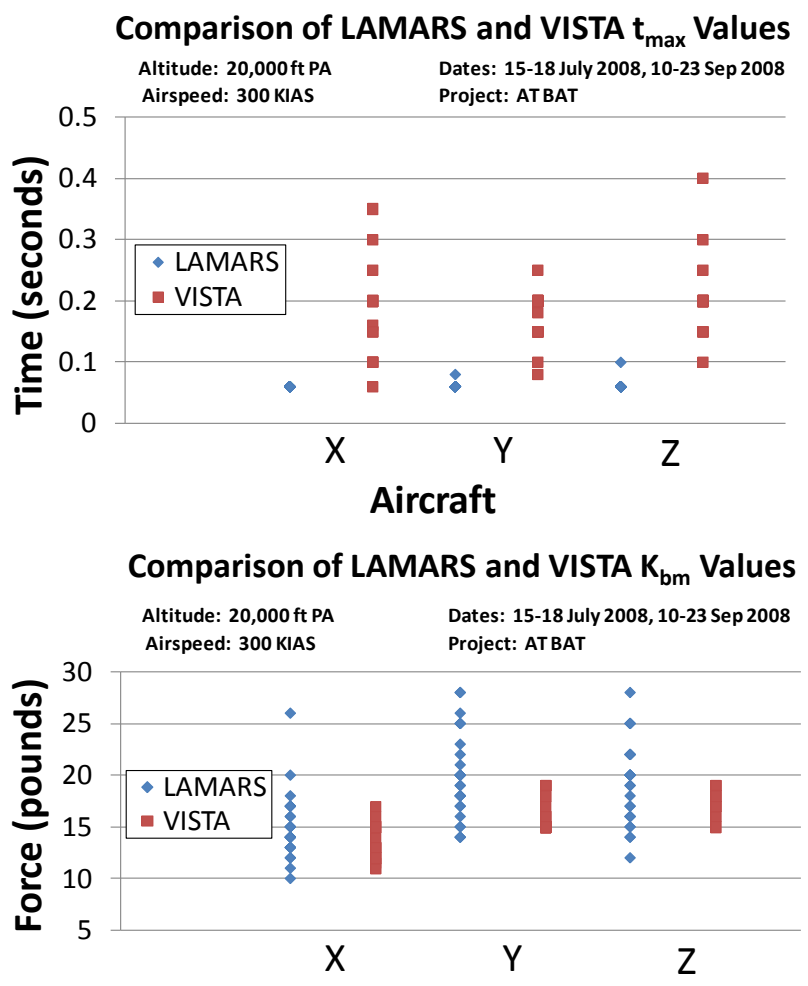

Aircraft

Figure 14: BAT Model Parameter Values

In summary, the following conclusions were made from observing pilot behavior in the presence of boundaries. Boundaries changed pilot behavior. As boundaries decreased, average pilot stick force, aggressiveness, and duty cycle increased, even though the tracking task did not change. Shrinking boundaries resulted in smaller roll error (i.e. better performance) until the boundaries became too small. At this point, the error (as a percentage of boundary size) began to increase. The proposed mathematical BAT model was in good agreement with pilot behavior during periods of boundary avoidance.

\section{Data Analysis: Cooper-Harper Ratings}

The second objective of the AT BAT program was to determine the correlation between performance and workload (quantitatively measured during a BAT plus secondary task profile) and the Cooper-Harper rating of a given aircraft (qualitatively assigned during a Cooper-Harper profile). BAT performance for a given aircraft model was defined as the average BAT task run time achieved with that model. (BAT performance could have also been defined as the minimum achievable boundary size. However, due to a small handling qualities spread among aircraft models in this test, this difference in BAT task run time was small, and each model was able to operate to the same minimum boundary size.) BAT workload was defined as the boundary size at which the average secondary task performance began to degrade. 
Histograms of Cooper-Harper ratings are shown in figure 15. As described in the discussion of aircraft model development, the handling qualities spread among the aircraft models was not as large as desired. All of the VISTA models and two of the LAMARS models received mostly Level 2 ratings. However, some small differences among the aircraft could be seen. For example, in VISTA both Aircraft X and Aircraft Y had median Cooper-Harper ratings of 4 (Level 2). However, Aircraft X received two Level 1 ratings, whereas Aircraft $Y$ did not receive any Level 1 ratings. Aircraft $Z$ had a median Cooper-Harper rating of 6. In LAMARS, the Aircraft X, Y, and Z had median Cooper-Harper ratings of 5, 6, and 7, respectively. Pilot comments confirmed that the handling qualities of each aircraft were in fact different.
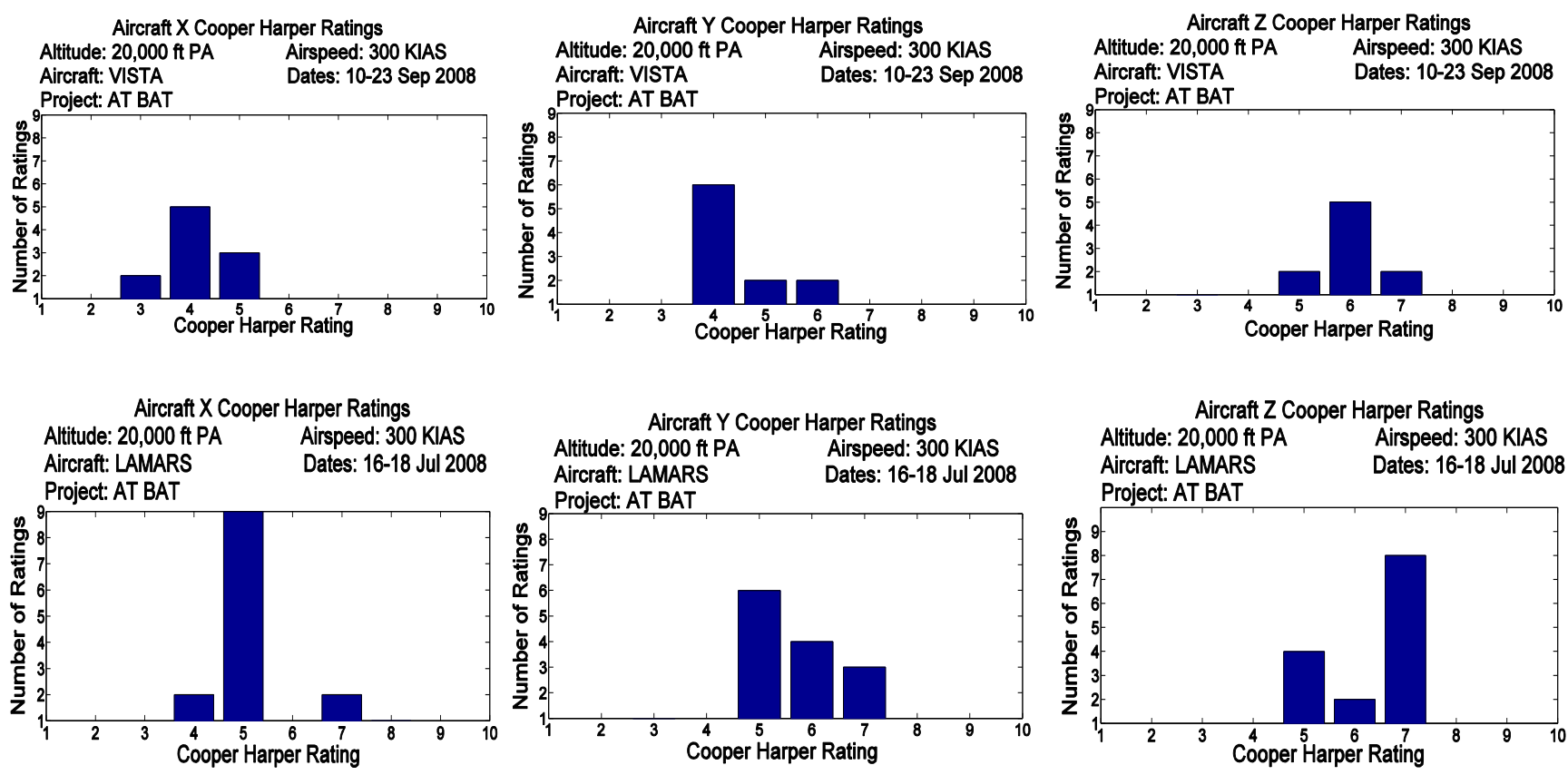

Figure 15: Cooper-Harper Rating Histograms

In figure 16 the median Cooper-Harper rating is displayed as a function of average BAT task run time. The error bars display the minimum and maximum Cooper-Harper ratings. In the LAMARS data, the average BAT run time was shortest for the aircraft with the worst handling qualities (Aircraft $Z$ ). Aircraft $X$ and $Y$ had very similar average BAT run times, which was not surprising given the small spread in Cooper-Harper ratings between the two. The VISTA data further supported this correlation. The average BAT run time was shortest for the aircraft with the worst handling qualities (Aircraft $\mathrm{Z}$ ) and longest for the aircraft with the best handling qualities (Aircraft X). The difference in average run time was small, however. Had the boundaries decreased in finer increments, it may have been possible to see an even larger spread in BAT run times to add further credence to this observation. BAT run time is a quantitative measure of task performance since a longer run time would indicate better performance by the pilot. The data portrayed in figure 16 indicated that a Cooper-Harper rating was indirectly proportional to the BAT run time, meaning a better rated aircraft (smaller Cooper-Harper rating) had a longer BAT run time. 

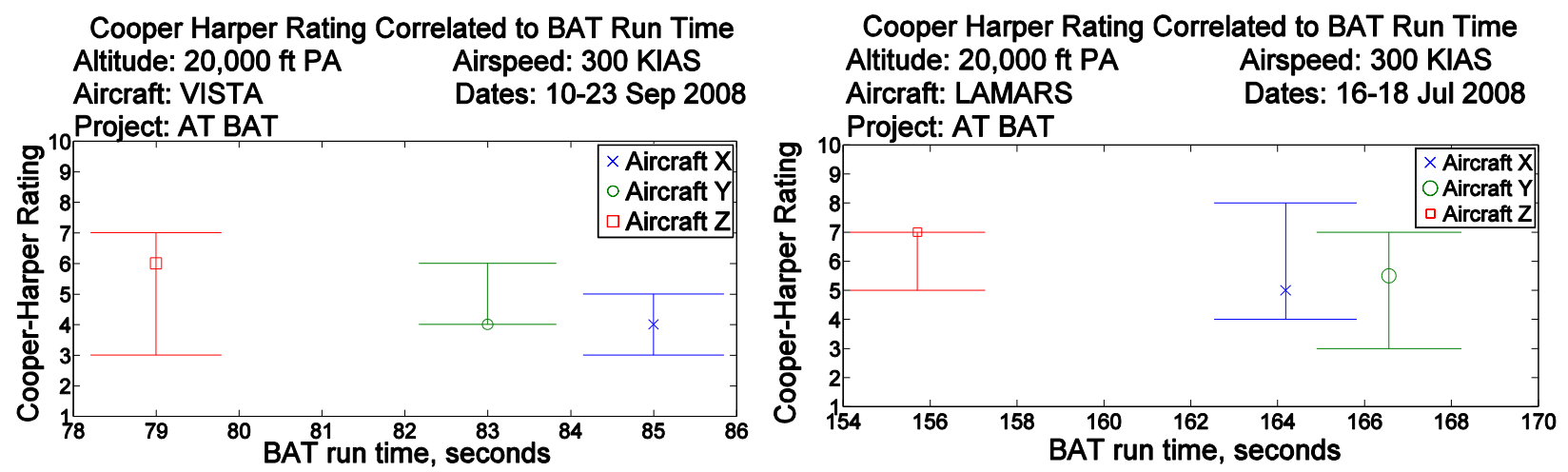

Figure 16: Cooper-Harper Ratings and Average BAT Task Run Time

Recall that the secondary task was used as a quantitative measure of workload. As expected, the average secondary task error increased with decreasing boundary size, because the pilot was devoting more attention to staying within the smaller boundaries than performing well on the secondary task. This correlation held true regardless of the aircraft handling qualities, but the point at which performance began to decrease varied with the aircraft handling qualities. For example, as shown in figure 17, aircraft $X$ in the VISTA, which had the best handling qualities, showed relatively constant secondary task error for the first two boundary sizes, and then a sudden increase in error when the boundaries shrank to \pm 16 degrees. Since the boundaries decreased step-wise, the exact boundary size at which increased error would occur (referred to as the critical boundary size) could not be determined. Therefore, a range of critical boundary sizes was given.
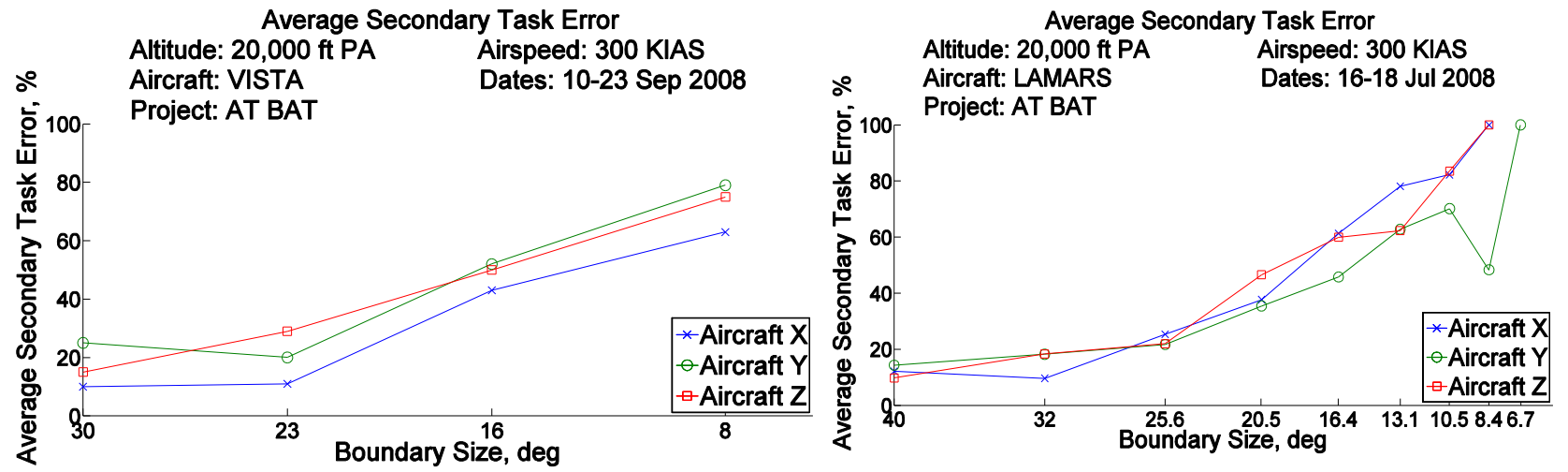

Figure 17: Average Secondary Task Error

Table 3 shows the critical boundary size ranges for each aircraft. In the VISTA, Aircraft $\mathrm{X}$ and $\mathrm{Y}$ had the same critical boundary size, probably due to the similarity in handling qualities. In LAMARS, Aircraft Y actually had a smaller critical boundary size than Aircraft X, even though the pilot opinion ratings were worse for Aircraft Y. It could be argued that the Aircraft $Y$ error was actually increasing from the start, and thus the critical boundary size for Aircraft $Y$ was greater than 32 degrees. This was almost definitely the case for Aircraft $Z$ in both the LAMARS and VISTA, in which the critical 
boundary size could not be directly determined, because the secondary task error increased linearly from the start of the task. The task would need to be started at a larger boundary size in order to determine the critical boundary size.

Table 3: Critical Boundary Size Ranges

\begin{tabular}{|c|c|c|}
\hline & LAMARS & VISTA \\
\hline Aircraft X & $25.6-32$ & $16-23$ \\
\hline Aircraft $Y$ & $20.5-25.6$ & $16-23$ \\
\hline Aircraft $Z$ & $>32$ & $>23$ \\
\hline
\end{tabular}

To more completely understand how the primary task affected the secondary task performance, the secondary task should be evaluated in isolation (i.e. without a primary task). In future tests, perform a secondary task profile with no primary task in order to provide a performance baseline. $\left(R 1^{1}\right)$

In general, the aircraft with better Cooper-Harper ratings had a smaller critical boundary size. Because an aircraft with better Cooper-Harper ratings had handling qualities that made the primary roll tracking task easier, the pilot could devote his attention to doing well on the secondary task, even in the presence of smaller boundaries. Secondary task performance began to degrade earlier (i.e. at larger boundaries) for aircraft with poor handling qualities. In this comparison, the data showed that the Cooper-Harper rating was proportional to the critical boundary size, meaning a better rated aircraft (smaller Cooper-Harper rating) had a smaller critical boundary size.

The following conclusions could therefore be made from comparing performance and workload (quantitatively measured during a BAT plus secondary task profile) with the Cooper-Harper rating of a given aircraft (qualitatively assigned during a Cooper-Harper profile). Aircraft with better (i.e. smaller) Cooper-Harper ratings tended to fly longer in the presence of boundaries than aircraft with worse (i.e. higher) CooperHarper ratings. Aircraft with better Cooper-Harper ratings tended to have degraded secondary task performance (i.e. increased workload and diminished spare capacity) at smaller boundary sizes than aircraft with worse Cooper-Harper ratings. The boundary size at which the secondary task performance degraded was called the critical boundary size.

In order for a Cooper-Harper rating to be relevant, the assigned task and the performance requirements were required to be operationally representative. Likewise, when using a BAT task to quantify pilot opinion, the given task and the requirements (desired and adequate minimum achievable and critical boundary sizes) must be suitable for the aircraft under test. For this test, suitable boundary sizes were determined through

\footnotetext{
${ }^{1}$ Numerals preceded by an $\mathrm{R}$ within parentheses at the end of a sentence correspond to the recommendation numbers tabulated in the Conclusions and Recommendations section of this report.
} 
trial and error during simulations and calibration flights. The amount of BAT flight test data was not sufficient to pre-define desired and adequate boundary sizes for all types of aircraft. Further BAT testing on aircraft with varying levels of handling qualities would allow these required boundary sizes to be determined for various aircraft classes and flight phase categories, much the same way as MIL-STD-1797B suitable flying qualities parameters were developed through historical data. Perform more testing with boundaries and secondary tasks in order to better quantify the relationship between subjective workload assessment and secondary task performance. (R2)

With adequately defined performance and workload criteria (in terms of desired and adequate minimum achievable and critical boundary sizes), a chart such as figure 18 could be built, in which the critical boundary size would be plotted against the minimum achievable boundary size. In this chart, the area in which the plotted point lay would correspond to the handling qualities level. Aircraft with a minimum achievable boundary size and a critical boundary size smaller than the desired sizes would be Level 1. Aircraft with either (or both) minimum achievable boundary size or a critical boundary size between the desired and adequate values would be Level 2. Aircraft with either (or both) minimum achievable boundary size or a critical boundary size between outside of the adequate values would be Level 3 .

Figure 18 plots the average results (discussed in the section above) for each aircraft model in the LAMARS and VISTA. The desired and adequate boundary sizes were chosen after testing so that the results corresponded with the assigned CooperHarper rating levels. As mentioned above, more testing (in order to establish a large database for various aircraft classes and flight phase categories,) would allow these values to be pre-determined. In the chart, all models but one fell in the Level 2 region. Aircraft $Z$ in LAMARS fell in the Level 3 region. This corresponded to the assigned Cooper-Harper ratings. 


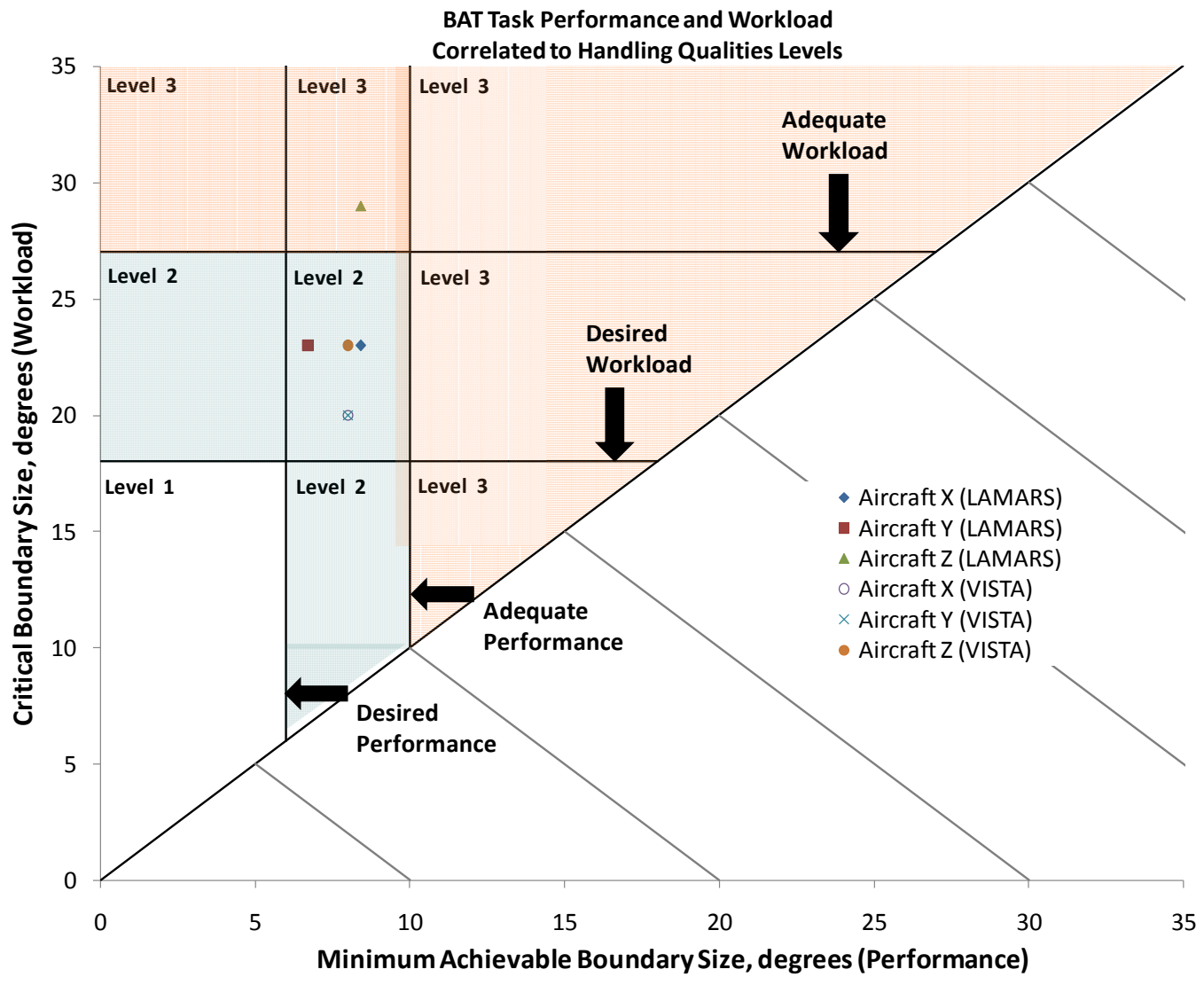

Figure 18: BAT Performance and Workload Correlated to Handling Qualities Levels 


\section{Conclusions and Recommendations}

The objective of the AT BAT program was to observe pilot behavior during a tracking task and secondary task in the presence of boundaries, and compare this behavior to the behavior predicted by the boundary avoidance tracking (BAT) theory. A second objective was to search for a correlation between the subjective pilot opinion ratings of the aircraft from a Cooper-Harper task and the objectively-measured performance and workload of a separate task in the roll axis. All objectives were met.

Boundaries changed pilot behavior. As boundaries decreased, average pilot stick force, aggressiveness, and duty cycle increased, even though the tracking task did not change. Shrinking boundaries resulted in smaller roll error (i.e. better performance) until the boundaries became too small. At this point, the error (as a percentage of boundary size) began to increase. The proposed mathematical BAT model qualitatively matched pilot behavior during periods of boundary avoidance.

Comparisons were made between performance and workload (quantitatively measured during a BAT plus secondary task profile) with the Cooper-Harper rating of a given aircraft (qualitatively assigned during a Cooper-Harper profile). Aircraft with better (i.e. smaller) Cooper-Harper ratings tended to fly longer in the presence of boundaries than aircraft with worse (i.e. higher) Cooper-Harper ratings. Due to a small handling qualities spread among aircraft models, however, this difference in BAT task run time was small. Aircraft with better Cooper-Harper ratings tended to have degraded secondary task performance (i.e. increased workload and diminished spare capacity) at smaller boundary sizes than aircraft with worse Cooper-Harper ratings. The boundary size at which the secondary task performance degraded was called the critical boundary size.

With adequately defined performance and workload criteria (in terms of desired and adequate minimum achievable and critical boundary sizes), critical boundary size could be plotted against the minimum achievable boundary size. The position of the plotted point would correspond to the handling qualities level. Aircraft with a minimum achievable boundary size and a critical boundary size smaller than the desired sizes would be Level 1 . Aircraft with either (or both) minimum achievable boundary size or a critical boundary size between the desired and adequate values would be Level 2 . Aircraft with either (or both) minimum achievable boundary size or a critical boundary size between outside of the adequate values would be Level 3 .

The following recommendations were made:

In future tests, perform a secondary task profile with no primary task in order to provide a performance baseline. $(\mathrm{R} 1, \mathrm{p.23})$

Perform more testing with boundaries and secondary tasks in order to determine desired and adequate minimum achievable and critical boundary sizes for various aircraft classes and flight phase categories. (R2, p. 24) 


\section{REFERENCES}

1. Gray, William. Boundary-Avoidance Tracking: A New Pilot Tracking Model. American Institute of Aeronautics and Astronautics, 2005.

2. Warren, Randy. An Investigation of the Effects of Boundary Avoidance on Pilot Tracking. Air Force Institute of Technology, 2006.

3. Dotter, Jason. An Analysis of Aircraft Handling Quality Data Obtained from Boundary Avoidance Tracking flight Test Techniques. Air Force Institute of Technology, 2007.

4. MIL-STD-1797B, Flying Qualities of Piloted Aircraft, 15 February 2006.

5. Gray, William. A Boundary Avoidance Tracking Flight Test Technique for Performance and Workload Assessment. Society of Experimental Test Pilots, 2007. 


\section{APPENDIX A - AIRCRAFT MODEL DESCRIPTIONS}

The aircraft models in LAMARS had the following characteristics:

Aircraft X: $\quad \frac{p}{\delta_{a}}=\frac{-29.15 s^{3}-47.07 s^{2}-242.5 s+0.4576}{s^{4}+3.81 s^{3}+12.64 s^{2}+18.13 s+0.181}$

$$
\begin{aligned}
& \omega_{d r}=3 \text { radians } / \text { second } \\
& \zeta_{d r}=0.3 \\
& \tau_{r}=0.5 \text { seconds } \\
& \tau_{s}=99 \text { seconds }
\end{aligned}
$$

Aircraft Y: $\quad \frac{p}{\delta_{a}}=\frac{-11.72 s^{3}-2.406 s^{2}-10.44 s+0.01966}{s^{4}+1.119 s^{3}+1.193 s^{2}+0.9209 s+0.009254}$

$$
\begin{aligned}
& \omega_{d r}=1 \text { radians } / \text { second } \\
& \zeta_{d r}=0.1 \\
& \tau_{r}=1.1 \text { seconds } \\
& \tau_{s}=98 \text { seconds }
\end{aligned}
$$

Aircraft Z: $\quad \frac{p}{\delta_{a}}=\frac{-6.152 s^{3}-0.2133 s^{2}-0.8743 s+0.001645}{s^{4}+0.526 s^{3}+0.1732 s^{2}+0.08168 s+0.0008033}$

$$
\begin{aligned}
& \omega_{d r}=0.4 \text { radians } / \text { second } \\
& \zeta_{d r}=0.02 \\
& \tau_{r}=2.5 \text { seconds } \\
& \tau_{s}=100 \text { seconds }
\end{aligned}
$$

Where:

$$
\begin{array}{ll}
p & =\text { roll rate } \\
\delta_{a} & =\text { aileron deflection (degrees) } \\
\omega_{d r} & =\text { Dutch roll frequency } \\
\zeta_{d r} & =\text { Dutch roll damping } \\
\tau_{r} & =\text { roll mode time constant } \\
\tau_{s} & =\text { spiral mode time constant }
\end{array}
$$


The roll mode and Dutch roll parameters were chosen from MIL-STD-1797B (reference 4) to correspond with Level 1, 2, and 3 handling qualities. Due to the continuously closed-loop nature of the roll tracking task in this project, the spiral mode was not considered a factor, and was not changed significantly among the models.

The stick force gradient was nonlinear, and was based on an F-16 gradient. Therefore, the transfer functions are presented here as a function of aileron input instead of stick force. Figure A-1 shows the step responses of each of the LAMARS aircraft models. The quick rise time and high Dutch roll damping are evident in the Aircraft $X$ response. The Aircraft $\mathrm{Y}$ and Aircraft $\mathrm{Z}$ responses showed progressively slower rise time, and lower Dutch roll frequency and damping.
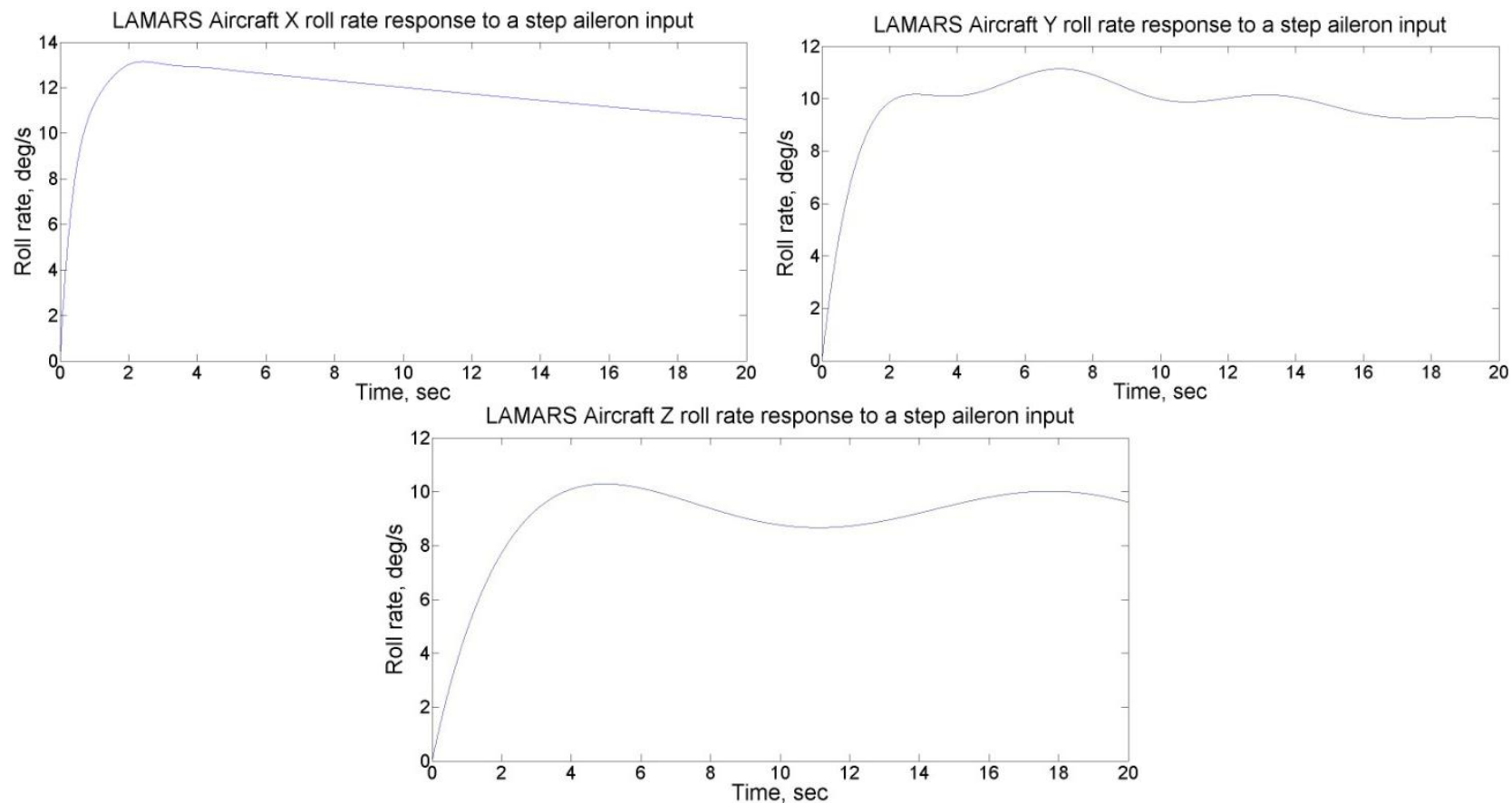

Figure A- 1: Roll rate response to aileron step, LAMARS

Note that all of the transfer functions contained a very slow non-minimum phase zero. Due to the chosen sign convention, this manifested itself in an initial response in the desired direction, followed by a very slow decay to a steady-state response in the opposite direction. Figure A-2 shows the Aircraft X long-term response. Aircraft Y and $\mathrm{Z}$ had similar long-term responses. Due to the very slow nature of this behavior, it was not noticed by the pilots. 


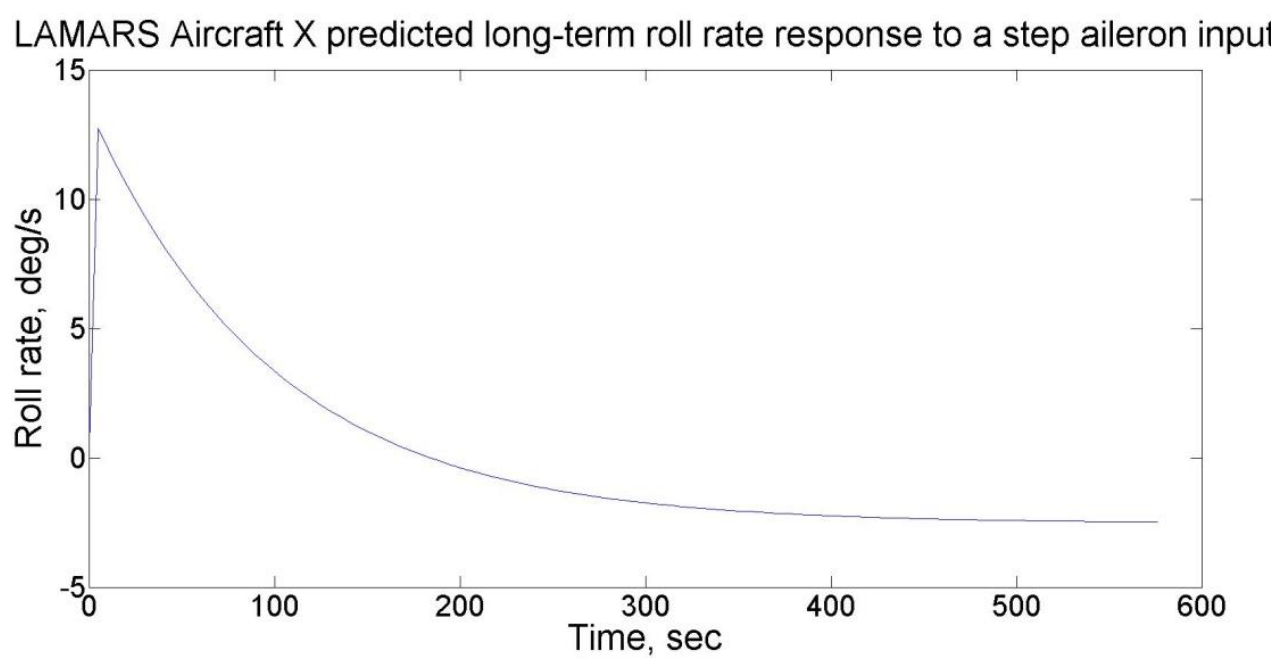

Figure A- 2: Long term roll rate response to aileron step, LAMARS

Because the handling qualities spread among the LAMARS models was less than desired, the models were changed for implementation in the VISTA. After several iterations (including results from the calibration sorties), the team decided to design each model with Level 1 Dutch roll characteristics, and use the roll mode time constant as the discriminator between the models. The spiral mode time constant was not intentionally changed, but it was allowed to change as necessary to appropriately model the roll mode in the VISTA. The following models were used for the VISTA flight test:

Aircraft X: $\quad \frac{p}{\delta_{a}}=\frac{-41.02 s^{3}-73.38 s^{2}-403.7 s+0.7464}{s^{4}+5.157 s^{3}+15.13 s^{2}+30.49 s+0.9795}$

$\omega_{d r}=3$ radians $/$ second

$\zeta_{d r}=0.3$

$\tau_{r}=0.3$ seconds

$\tau_{s}=31$ seconds

Aircraft Y: $\quad \frac{p}{\delta_{a}}=\frac{-13.05 s^{3}-26.96 s^{2}-122.1 s+0.2252}{s^{4}+2.722 s^{3}+10.68 s^{2}+8.424 s+0.1631}$

$\omega_{d r}=3$ radians $/$ second

$\zeta_{d r}=0.3$

$\tau_{r}=1.1$ seconds

$\tau_{s}=50$ seconds 
Aircraft Z: $\quad \frac{p}{\delta_{a}}=\frac{-10.31 s^{3}-17.06 s^{2}-95.53 s+0.1773}{s^{4}+2.213 s^{3}+9.753 s^{2}+3.804 s-0.00801}$

$$
\begin{aligned}
& \omega_{d r}=3 \text { radians } / \text { second } \\
& \zeta_{d r}=0.3 \\
& \tau_{r}=2.4 \text { seconds } \\
& t_{d}=380 \text { seconds (time to double amplitude) }
\end{aligned}
$$

Figure A-3 shows the predicted step responses of each of the VISTA aircraft models. The quick rise time is evident in the Aircraft X response. The Aircraft $\mathrm{Y}$ and Aircraft $Z$ responses show progressively slower rise time. All models exhibited acceptable Dutch roll parameters. Note that the predicted spiral mode for Aircraft $Z$ was unstable. The VISTA predicted models also displayed the same non-minimum phase behavior as the LAMARS models.
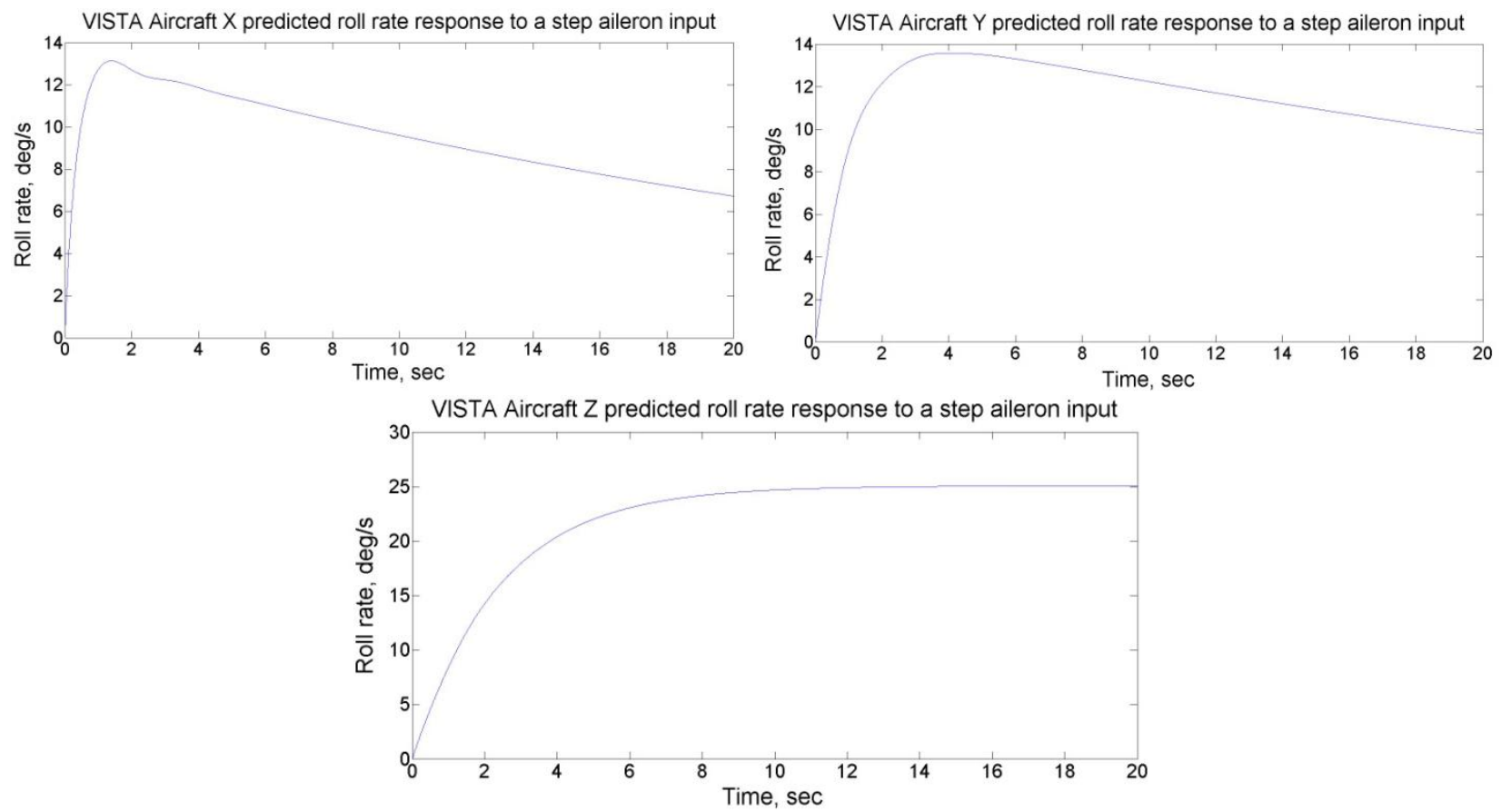

Figure A- 3: Predicted roll rate response to aileron step, VISTA

During flight test, lateral step inputs were performed in order to validate the predicted response displayed in figure A-3. The step inputs were performed with lateral stick inputs instead of pure aileron inputs. Since the stick force gradient was nonlinear, it couldn't be adequately modeled with a transfer function. Therefore, the predicted aileron-to-roll-rate response and the flight test stick-to-roll-rate response can't be directly compared. However, the results can be compared qualitatively and are presented in 
figure A-4. Note that the roll mode time constants are significantly higher than predicted. In fact, the Aircraft $Y$ response never reached steady state (with a 3 second input). The Aircraft $\mathrm{Z}$ response was unstable, with a much larger time-to-double amplitude than predicted.

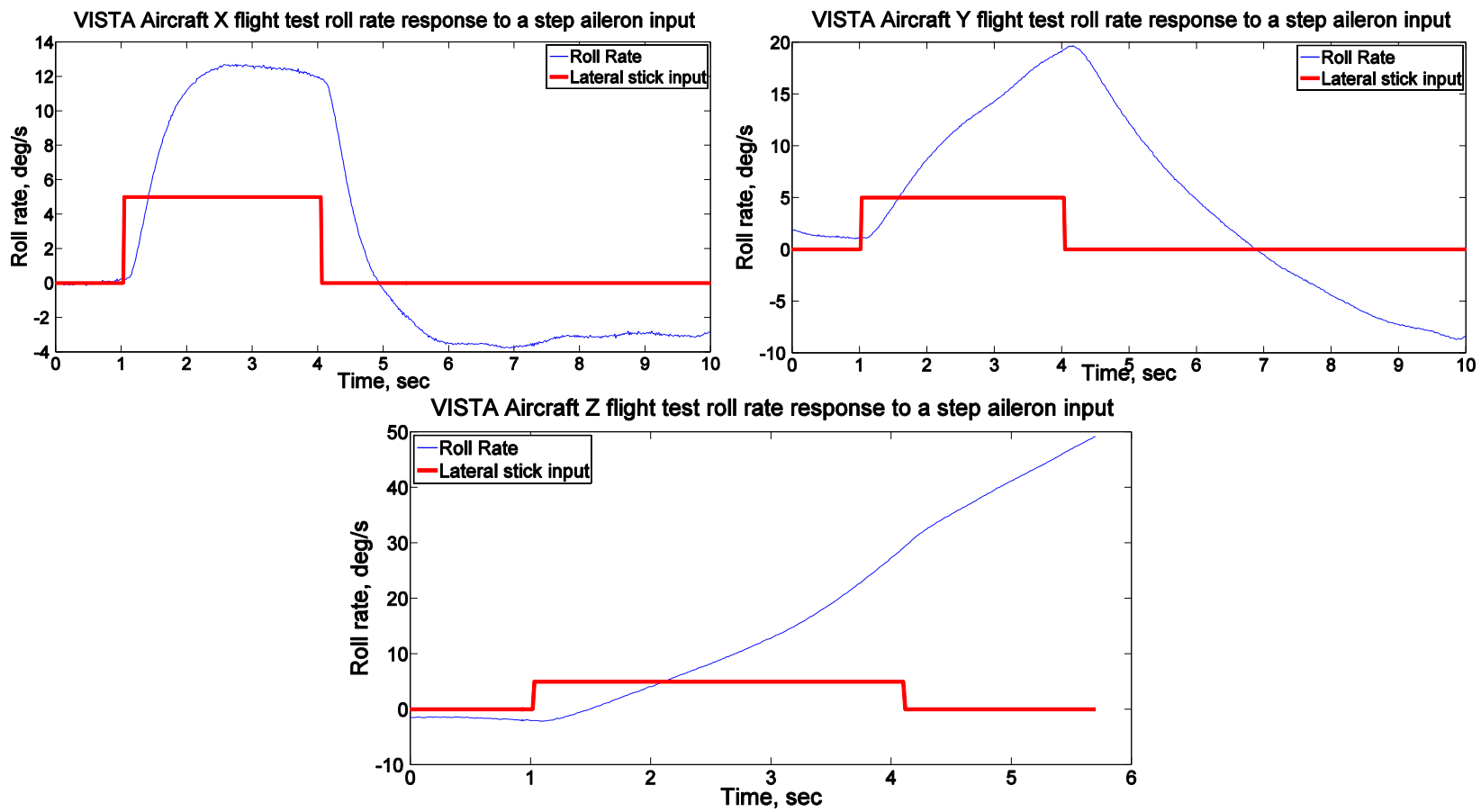

Figure A- 4: Flight test roll rate response to aileron step, VISTA

In summary, the actual aircraft characteristics were not as predicted, but were suitable for flight test use. 


\section{APPENDIX B - COMPLETED TEST POINT MATRIX}

Table 4: Completed Test Point Matrix

\begin{tabular}{|c|c|c|c|}
\hline Test & Pilot & Profile & Aircraft Model \\
\hline 1 & 1 & BAT Plus & $x$ \\
\hline 2 & 1 & BAT & $Y$ \\
\hline 3 & 1 & BAT Plus & 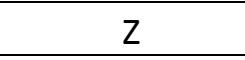 \\
\hline 4 & 1 & $\mathrm{CHR}$ & $Y$ \\
\hline 5 & 1 & BAT & $x$ \\
\hline 6 & 1 & $\mathrm{CHR}$ & Z \\
\hline 7 & 1 & BAT Plus & $Y$ \\
\hline 8 & 1 & BAT & Z \\
\hline 9 & 1 & $\mathrm{CHR}$ & $x$ \\
\hline 10 & 2 & $\mathrm{CHR}$ & $Y$ \\
\hline 11 & 2 & BAT & $x$ \\
\hline 12 & 2 & $\mathrm{CHR}$ & Z \\
\hline 13 & 2 & BAT Plus & $x$ \\
\hline 14 & 2 & BAT & $Y$ \\
\hline 15 & 2 & BAT Plus & Z \\
\hline 16 & 2 & $\mathrm{CHR}$ & $x$ \\
\hline 17 & 2 & BAT & Z \\
\hline 18 & 2 & BAT Plus & $Y$ \\
\hline 19 & 3 & BAT & $x$ \\
\hline 20 & 3 & $\mathrm{CHR}$ & $Y$ \\
\hline 21 & 3 & BAT & Z \\
\hline 22 & 3 & BAT Plus & $Y$ \\
\hline 23 & 3 & $\mathrm{CHR}$ & $x$ \\
\hline 24 & 3 & BAT Plus & Z \\
\hline 25 & 3 & BAT & $Y$ \\
\hline 26 & 3 & $\mathrm{CHR}$ & Z \\
\hline 27 & 3 & BAT Plus & $\mathrm{x}$ \\
\hline 28 & 4 & BAT Plus & $\mathrm{x}$ \\
\hline 29 & 4 & BAT & $Y$ \\
\hline 30 & 4 & BAT Plus & Z \\
\hline 31 & 4 & $\mathrm{CHR}$ & $Y$ \\
\hline 32 & 4 & BAT & $x$ \\
\hline 33 & 4 & $\mathrm{CHR}$ & Z \\
\hline 34 & 4 & BAT Plus & $Y$ \\
\hline 35 & 4 & BAT & Z \\
\hline
\end{tabular}




\begin{tabular}{|c|c|c|c|}
\hline Test & Pilot & Profile & Aircraft Model \\
\hline 36 & 4 & $\mathrm{CHR}$ & $x$ \\
\hline 37 & 5 & BAT & Z \\
\hline 38 & 5 & BAT Plus & $x$ \\
\hline 39 & 5 & BAT & $Y$ \\
\hline 40 & 5 & $\mathrm{CHR}$ & $x$ \\
\hline 41 & 5 & BAT Plus & Z \\
\hline 42 & 5 & $\mathrm{CHR}$ & $Y$ \\
\hline 43 & 5 & BAT & $x$ \\
\hline 44 & 5 & BAT Plus & $Y$ \\
\hline 45 & 5 & $\mathrm{CHR}$ & Z \\
\hline 46 & 1 & BAT Plus & $x$ \\
\hline 47 & 1 & $\mathrm{CHR}$ & Z \\
\hline 48 & 1 & BAT Plus & $Y$ \\
\hline 49 & 1 & BAT & Z \\
\hline 50 & 1 & $\mathrm{CHR}$ & $x$ \\
\hline 51 & 1 & BAT & $Y$ \\
\hline 52 & 1 & BAT Plus & Z \\
\hline 53 & 1 & $\mathrm{CHR}$ & $Y$ \\
\hline 54 & 1 & BAT & $x$ \\
\hline 55 & 2 & BAT & $Y$ \\
\hline 56 & 2 & BAT Plus & $\mathrm{x}$ \\
\hline 57 & 2 & BAT & Z \\
\hline 58 & 2 & $\mathrm{CHR}$ & $x$ \\
\hline 59 & 2 & BAT Plus & $Y$ \\
\hline 60 & 2 & $\mathrm{CHR}$ & Z \\
\hline 61 & 2 & BAT & $x$ \\
\hline 62 & 2 & BAT Plus & Z \\
\hline 63 & 2 & $\mathrm{CHR}$ & $Y$ \\
\hline 64 & 3 & BAT & $\mathrm{x}$ \\
\hline 65 & 3 & BAT Plus & $Y$ \\
\hline 66 & 3 & BAT & Z \\
\hline 67 & 3 & $\mathrm{CHR}$ & $Y$ \\
\hline 68 & 3 & BAT Plus & $x$ \\
\hline 69 & 3 & $\mathrm{CHR}$ & Z \\
\hline 70 & 3 & BAT & $Y$ \\
\hline 71 & 3 & BAT Plus & $z$ \\
\hline 72 & 3 & $\mathrm{CHR}$ & $x$ \\
\hline 73 & 4 & BAT & Z \\
\hline 74 & 4 & $\mathrm{CHR}$ & $x$ \\
\hline
\end{tabular}

B-2 


\begin{tabular}{|c|c|c|c|}
\hline Test & Pilot & Profile & Aircraft Model \\
\hline 75 & 4 & BAT & Y \\
\hline 76 & 4 & BAT Plus & X \\
\hline 77 & 4 & CHR & Z \\
\hline 78 & 4 & BAT Plus & Y \\
\hline 79 & 4 & BAT & Y \\
\hline 80 & 4 & CHR & Z \\
\hline 81 & 4 & BAT Plus & Y \\
\hline 82 & 5 & CHR & X \\
\hline 83 & 5 & BAT & Z \\
\hline 84 & 5 & CHR & Y \\
\hline 85 & 5 & BAT Plus & Z \\
\hline 86 & 5 & BAT & X \\
\hline 87 & 5 & BAT Plus & Z \\
\hline 88 & 5 & CHR & Y \\
\hline 89 & 5 & BAT & \\
\hline 90 & 5 & BAT Plus & \\
\hline
\end{tabular}

CHR - Roll-angle tracking task with no boundaries or secondary task BAT - Roll-angle tracking task with boundaries but no secondary task BAT Plus - Roll-angle tracking task with boundaries and a secondary task 


\section{APPENDIX C - COOPER-HARPER RATING SCALE}

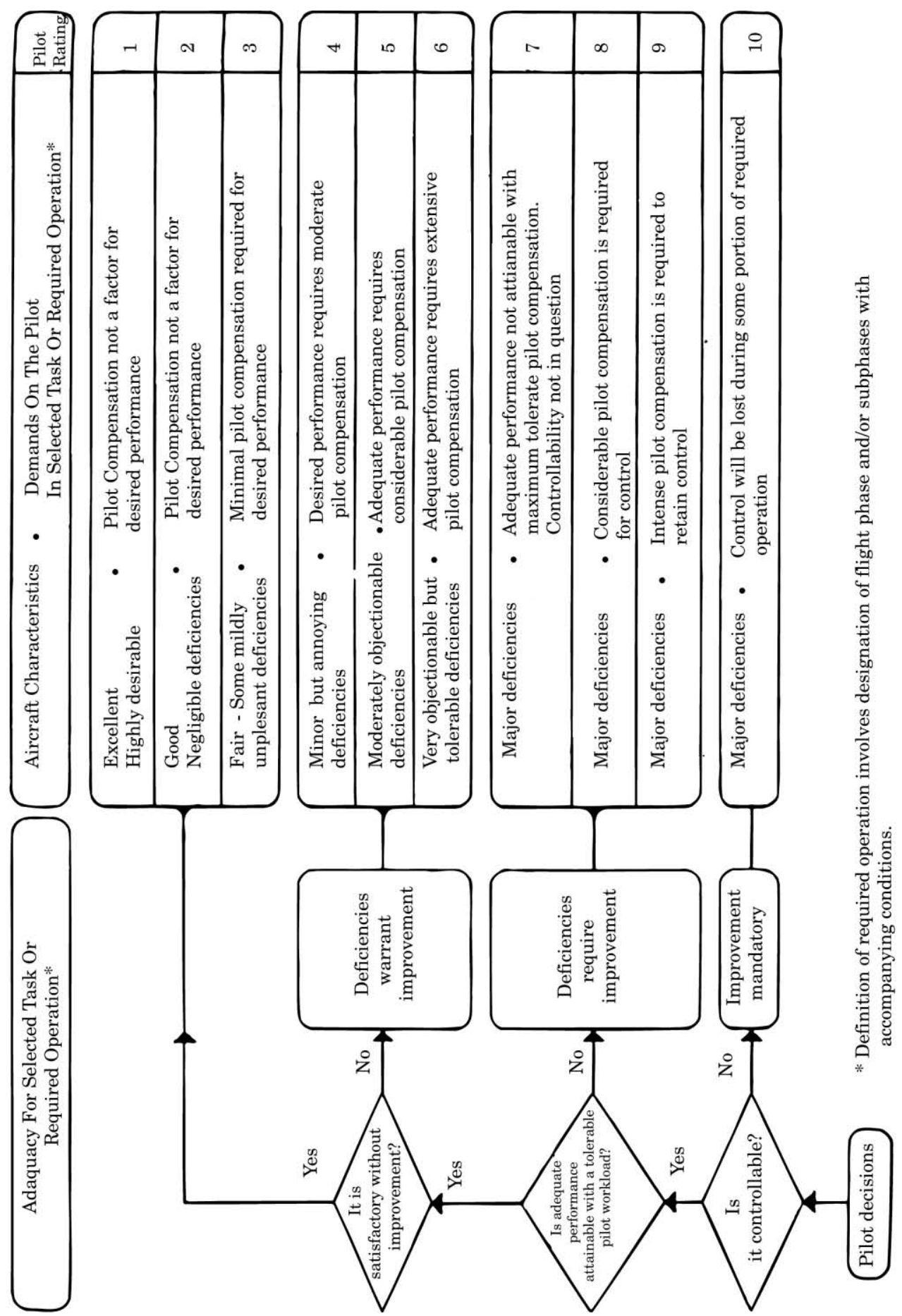

C-1 


\section{APPENDIX D - ACRONYM LIST}

\section{$\underline{\text { Symbol Definition }}$}

Units

AFFTC Air Force Flight Test Center

AFIT

Air Force Institute of Technology

BAT

CCD

boundary avoidance tracking

CHR

charge-coupled device

EP

Cooper-Harper rating

FCP

evaluation pilot

$-$

HUD

front cockpit

$-$

LAMARS

heads-up display

PIO

RCP

Large Amplitude Multi-Mode Aerospace Research Simulator

$--$

pilot-in-the-loop oscillation

rear cockpit

SP

safety pilot

TMP

Test Management Project

TPS

U.S. Air Force Test Pilot School

USAF

United States Air Force

VISTA

Variable-Stability In-Flight Simulator Test Aircraft

VSS

variable stability system 
The following identifies the distribution of AT BAT technical reports. All reports will be distributed by the $412 \mathrm{TW} / \mathrm{DOC}$.

\section{AFFTC/HO}

305 E Popson Ave, Bldg 1405

Edwards AFB CA 93524-6595

USAF TPS/EDT

Attn: Mr Gary Aldrich

$220 \mathrm{~S}$ Wolfe Ave, Bldg 1220

Edwards AFB CA 93524-6485

812 TSS/ENTL

307 E Popson Ave, Bldg 1400

Edwards AFB CA 93524-6630

USAF TPS/EDC

Attn: Ms Dottie Meyer

220 S Wolfe Ave, Bldg 1220

Edwards AFB CA 93524-6485

For "offsite" distribution:

Defense Technical Information Center (DTIC)

8725 John J. Kingman Rd, Ste 0944

ATTN: Willis Smith (DTIC-OCA)

Fort Belvoir, VA 22060-6218

AFIT Academic Library

ATTN: Librarian

Bldg 642

2950 Hobson Way

Wright Patterson AFB, OH 45433-7765

Maj Ryan Blake

16 Columbia Court

Edwards, CA 93523 